JOURNAL OF THE

AMERICAN MATHEMATICAL SOCIETY

Volume 10, Number 4, October 1997, Pages 939-972

S 0894-0347(97)00245-2

\title{
INTEGRAL TRANSFORMS WITH EXPONENTIAL KERNELS AND LAPLACE TRANSFORM
}

\author{
MASAKI KASHIWARA AND PIERRE SCHAPIRA
}

\section{INTRODUCTION}

An integral transform associates to each section of some sheaf on a manifold $X$ a section of another sheaf on a manifold $Y$, by a formula like:

$$
u \mapsto v=\int_{g} f^{*}(u) k,
$$

where $k$ is a kernel defined on a third manifold $Z$ (usually $Z=X \times Y$ ) and we have two morphisms

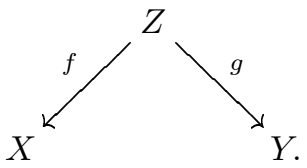

The language of sheaves and $\mathcal{D}$-modules is well adapted to this situation and many classical results may be interpreted in this framework. In the language of sheaves, equation (1.1) should be read as:

$$
F \mapsto F \circ K=g_{!}\left(f^{-1} F \otimes K\right),
$$

where $F$ is a sheaf on $X, K$ is a sheaf on $Z$, and $g_{!}, f^{-1}$ and $\otimes$ are the usual operations in the derived categories of sheaves. In the case of $\mathcal{D}$-modules (on complex manifolds) there is a similar construction, using the operations on $\mathcal{D}$ modules. When combining both languages one gets a nice adjunction formula which asserts, roughly speaking, that if $F$ is a sheaf on $X$ and $\mathcal{N}$ a $\mathcal{D}$-module on $Y$, $\mathcal{K}$ a regular holonomic $\mathcal{D}$-module on $Z$ and $K$ the associated perverse sheaf, then there is a natural isomorphism between the complex of holomorphic solutions of $(F \circ K) \otimes \mathcal{N}$ on $Y$ and the complex of holomorphic solutions of $F \otimes(\mathcal{K} \circ \mathcal{N})$ on $X$ (see [D'A-S1], [D'A-S2], [K-S2], [K-Sm]).

Our aim in this paper is the study of the Laplace transform. If $V$ is an $n$ dimensional complex vector space (more generally, a complex vector bundle) and $V^{*}$ its dual, this transform is described by the formula:

$$
u(z) \mapsto \int \exp (\langle z, w\rangle) u(z) d z .
$$

If we want to interpret this formula in the framework described above, a serious difficulty appears: the $\mathcal{D}$-module generated by the kernel $\exp (\langle z, w\rangle)$ on $P \times P^{*}$, the projective compactification of $V \times V^{*}$, is holonomic, but not regular. In fact, this is

Received by the editors September 17, 1996 and, in revised form, May 23, 1997.

1991 Mathematics Subject Classification. Primary 32C38, 14F10, 44A10. 
the reason why Fourier transform does not apply to hyperfunctions nor distributions on a real vector space, and why one has to consider tempered distributions or rapidly decreasing functions.

Fortunately, the functorial analogue of such distributions or functions already exists: these are the functors $\operatorname{Thom}(\cdot, \mathcal{O})$ of moderate cohomology and the functor $\cdot \stackrel{w}{\otimes} \mathcal{O}$ of formal cohomology, introduced in $[\mathrm{K}]$ and $[\mathrm{K}-\mathrm{S} 2]$, respectively.

Hence we begin by making a study of general integral transforms in the situation (1.2) associated to a kernel

$$
\mathcal{L}=\left(\mathcal{D}_{Z} \exp (\varphi)\right)(* S)
$$

where $\varphi$ is a meromorphic function on $Z$ with poles in $S \subset Z$. Our main result (Proposition 4.2.1) are adjunction formulas in this context.

Coming back to the Laplace transform, let us denote by $j: V \hookrightarrow P$ the projective compactification of the vector space $V$. If $F$ is $\mathbb{R}$-constructible and $\mathbb{R}^{+}$-conic on $V$, set for short:

$$
\begin{aligned}
& F \stackrel{W}{\otimes} \mathcal{O}_{V}=\mathrm{R} \Gamma\left(P ; j ! F^{\otimes} \mathcal{O}_{P}\right), \\
& \operatorname{THom}\left(F, \mathcal{O}_{V}\right)=\mathrm{R} \Gamma\left(P ; \operatorname{Thom}\left(j_{!} F, \mathcal{O}_{P}\right)\right) .
\end{aligned}
$$

As a particular case of our adjunction formulas we obtain the Laplace isomorphisms

$$
\begin{aligned}
& L: F \stackrel{W}{\otimes} \mathcal{O}_{V} \stackrel{\sim}{\longrightarrow} F^{\wedge}[n] \stackrel{W}{\otimes} \mathcal{O}_{V^{*}}, \\
& { }^{t} L: \operatorname{THom}\left(F, \mathcal{O}_{V}\right) \stackrel{\sim}{\sim} \operatorname{THom}\left(F^{\wedge}[n], \mathcal{O}_{V^{*}}\right)
\end{aligned}
$$

where $F^{\wedge}$ denotes the Fourier-Sato transform of $F$. Moreover these isomorphisms are linear over the Weyl algebra $D(V)$ (identifying $D\left(V^{*}\right)$ with $D(V)$ by the Fourier transform), and admit inverses, associated with the kernel $\exp (-\langle z, w\rangle)$.

We discuss some applications of these formulas.

a) Let $U$ be an open convex subanalytic cone in $V$, and set

$$
Z=U^{\circ a}=\left\{w \in V^{*} ; \operatorname{Re}\langle z, w\rangle \leq 0\right\} .
$$

We get the isomorphism

$$
\mathrm{R} \Gamma_{[U]}\left(V ; \mathcal{O}_{V}\right) \simeq \mathrm{R} \Gamma_{[Z]}\left(V^{*} ; \mathcal{O}_{V^{*}}\right)[n]
$$

where we have set, for a locally closed subanalytic cone $S$,

$$
\mathrm{R} \Gamma_{[S]}\left(V ; \mathcal{O}_{V}\right)=\operatorname{THom}\left(\mathbb{C}_{S}, \mathcal{O}_{V}\right) .
$$

Both sides of (1.9) are concentrated in degree 0. If $V=\mathbb{C} \otimes_{\mathbb{R}} V_{\mathbb{R}}$ is the complexification of a real vector space $V_{\mathbb{R}}$ and $U=U^{\prime} \oplus \sqrt{-1} V_{\mathbb{R}}$ is an open convex tube, one recovers a well-known result, since the right hand side of (1.9) is the space $\Gamma_{Z}\left(\mathcal{S}^{\prime}\left(V_{\mathbb{R}}^{*}\right)\right)$ of Schwartz's tempered distributions on $V_{\mathbb{R}}^{*}$ supported by $Z$, and the left hand side is the space of holomorphic functions on $U$ with tempered growth on the boundary (including infinity).

b) If $\lambda$ is a non-degenerate quadratic solid cone in $V_{\mathbb{R}}$ with $p$ positive eigenvalues and if $\lambda^{\circ}$ denotes the closed tube associated with the dual cone, we find the isomorphism:

$$
\Gamma_{\lambda}\left(\mathcal{S}^{\prime}\left(V_{\mathbb{R}}\right)\right) \simeq H_{\left[\lambda^{\circ}\right]}^{p}\left(V^{*} ; \mathcal{O}_{V^{*}}\right)
$$

(a situation already considered by $[\mathrm{F}-\mathrm{G}]$ ). 
c) In $\S 6.3$, we introduce the conic sheaf $\mathcal{O}_{V}^{t}$ associated with the presheaf $U \mapsto$ THom $\left(\mathbb{C}_{U}, \mathcal{O}_{V}\right)$, and we show that the Laplace transform induces an isomorphism of conic sheaves:

$$
\left(\mathcal{O}_{V}^{t}\right)^{\wedge}[n] \simeq \mathcal{O}_{V^{*}}^{t}
$$

As a corollary, we recover the result of $[\mathrm{B}-\mathrm{M}-\mathrm{V}]$ and $[\mathrm{H}-\mathrm{K}]$ : if $M$ is a monodromic module over the Weyl algebra $D(V)$ and $M^{\vee}$ denotes the $D\left(V^{*}\right)$-module obtained by Fourier transform, then there is a natural isomorphism of conic sheaves

$$
\mathrm{R} \mathcal{H o m}_{D(V)}\left(M, \mathcal{O}_{V}\right)^{\wedge}[n] \simeq \mathrm{R} \mathcal{H} \operatorname{com}_{D\left(V^{*}\right)}\left(M^{\vee}, \mathcal{O}_{V^{*}}\right) .
$$

d) Let $\Omega=\left\{x \in M_{n}(\mathbb{C}) ; x\right.$ is symmetric and $\operatorname{Re} x$ is positive definite $\}$. The integral

$$
u(t) \mapsto v(x)=\int_{\mathbb{R}^{n}} e^{-\langle t, x t\rangle} u(t) d t
$$

allows us to identify $\mathcal{S}^{\prime}\left(\mathbb{R}^{n}\right)$ with the space of tempered holomorphic functions on $\Omega$ satisfying some system of differential equations that we calculate explicitly. This is an interpretation of the embedding of the Weil representation into the degenerate principal series.

\section{Notations AND REVIEW}

2.1. Notations. We refer to [K-S1] for an exposition of the sheaf theory, and we shall mainly follow the notations of this book.

If $X$ is a topological space, we denote by $\mathrm{D}^{\mathrm{b}}\left(\mathbb{C}_{X}\right)$ the bounded derived category of sheaves of $\mathbb{C}$-vector spaces on $X$. We denote as usual by $f^{-1}, f^{!}, \mathrm{R} f_{!}, \mathrm{R} f_{*}$, $\mathrm{R} \mathcal{H}$ om and $\otimes$ the six operations on sheaves of $\mathbb{C}$-vector spaces, and we set:

$$
D_{X}^{\prime}(\cdot)=\mathrm{R} \mathcal{H o m}\left(\cdot, \mathbb{C}_{X}\right) \text {. }
$$

If $\tau: V \rightarrow X$ is a real vector bundle, we denote by $\mathrm{D}_{\mathbb{R}^{+}}^{\mathrm{b}}\left(\mathbb{C}_{V}\right)$ the full subcategory of $\mathrm{D}^{\mathrm{b}}\left(\mathbb{C}_{V}\right)$ consisting of objects $F$ such that $H^{j}(F)$ is locally constant on the $\mathbb{R}^{+}$-orbits for all $j$. We will recall later the construction of the Fourier-Sato transform.

Now assume that $X$ is real analytic. We denote by $\mathbb{R}$-cons $\left(\mathbb{C}_{X}\right)$ the abelian category of $\mathbb{R}$-constructible sheaves of $\mathbb{C}$-vector spaces on $X$. By a result of $[\mathrm{K}]$, its bounded derived category is equivalent to $D_{\mathbb{R}-c}^{b}\left(\mathbb{C}_{X}\right)$, the full subcategory of $\mathrm{D}^{\mathrm{b}}\left(\mathbb{C}_{X}\right)$ consisting of objects $F$ with $H^{j}(F)$ in $\mathbb{R}$-cons $\left(\mathbb{C}_{X}\right)$ for all $j$.

If $\tau: V \rightarrow X$ is a real vector bundle over $X$, we set $\mathrm{D}_{\mathbb{R}^{+}, \mathbb{R}^{-c}}^{\mathrm{b}}\left(\mathbb{C}_{V}\right)=\mathrm{D}_{\mathbb{R}^{+}}^{\mathrm{b}}\left(\mathbb{C}_{V}\right) \cap$ $\mathrm{D}_{\mathbb{R}-\mathrm{c}}^{\mathrm{b}}\left(\mathbb{C}_{V}\right)$.

On a real analytic manifold $X$, we shall encounter the sheaves:

- $\mathcal{A}_{X}$ : the sheaf of real analytic functions,

- $\mathcal{C}_{X}^{\infty}$ : the sheaf of $\mathrm{C}^{\infty}$-functions,

- $\mathcal{D} b_{X}$ : the sheaf of distributions,

- $\mathcal{B}_{X}$ : the sheaf of hyperfunctions.

Let $A$ be a sheaf of rings on $X$. We denote by $A^{\text {opp }}$ the ring $A$ with the opposite multiplication rule. An $A$-module will mean a left $A$-module. Hence an $A^{\text {opp }}$ module is a right $A$-module. We denote by $\mathrm{D}^{\mathrm{b}}(A)$ the bounded derived category of sheaves of $A$-modules.

Now let $X$ be a complex manifold, $\mathcal{O}_{X}$ its structural sheaf, and $d_{X}$ its complex dimension. We denote by $\Omega_{X}^{p}$ the sheaf of holomorphic $p$-forms on $X$, and we set 
$\Omega_{X}=\Omega_{X}^{d_{X}}$. We denote by $\mathcal{D}_{X}$ the sheaf of rings of finite-order differential operators on $X$.

If $Z$ is a smooth submanifold of codimension $d$ in $X$, recall that one denotes by $\mathcal{B}_{Z \mid X}$ the regular holonomic $\mathcal{D}_{X}$-module $H_{[Z]}^{d}\left(\mathcal{O}_{X}\right)$.

If $\mathcal{M}$ is a $\mathcal{D}_{X}$-module, we set for short:

$$
\operatorname{Sol}(\mathcal{M})=\mathrm{R} \mathcal{H} \operatorname{om}_{\mathcal{D}_{X}}\left(\mathcal{M}, \mathcal{O}_{X}\right) .
$$

We denote by $\mathrm{D}_{\mathrm{q}-\text { good }}^{\mathrm{b}}\left(\mathcal{D}_{X}\right)$ the full triangulated subcategory of $\mathrm{D}^{\mathrm{b}}\left(\mathcal{D}_{X}\right)$ consisting of objects $\mathcal{M}$ such that $H^{j}(\mathcal{M})$ is quasi-good for all $j$. Here, an $\mathcal{O}_{X}$-module $\mathcal{F}$ is called quasi-good if any compact subset of $X$ has a neighborhood $U$ such that $\left.\mathcal{F}\right|_{U}$ is a union of an increasing countable family of coherent $\left.\mathcal{O}_{X}\right|_{U}$-submodules. A $\mathcal{D}_{X}$-module $\mathcal{M}$ is quasi-good if it is quasi-good as an $\mathcal{O}_{X}$-module. This definition coincides with the one given in [K-S2] by the following lemma.

Lemma 2.1.1. The subcategory of quasi-good $\mathcal{O}_{X}$-modules is closed under extensions, kernels and cokernels.

Proof. It is easy to see that this subcategory is closed under kernels and cokernels. Let us show that if $0 \rightarrow \mathcal{F}^{\prime} \stackrel{f}{\rightarrow} \mathcal{F} \stackrel{g}{\rightarrow} \mathcal{F}^{\prime \prime} \rightarrow 0$ is an exact sequence of $\mathcal{O}_{X}$-modules and if $\mathcal{F}^{\prime}$ and $\mathcal{F}^{\prime \prime}$ are quasi-good, then so is $\mathcal{F}$. Since the union of an increasing sequence of quasi-good submodules is quasi-good, we may assume from the beginning that $\mathcal{F}^{\prime \prime}$ is coherent. For a compact set $K$ of $X$, take finitely many open subsets $U_{i}$ of $X$ and a locally finitely generated submodule $\mathcal{G}_{i}$ of $\left.\mathcal{F}\right|_{U_{i}}$ such that $K \subset U=\bigcup_{i} U_{i}$ and $g\left(\mathcal{G}_{i}\right)=\left.\mathcal{F}^{\prime \prime}\right|_{U_{i}}$. Since $\left.0 \rightarrow \mathcal{G}_{i} \cap \operatorname{Ker} g \rightarrow \mathcal{G}_{i} \rightarrow \mathcal{F}^{\prime \prime}\right|_{U_{i}} \rightarrow 0$ is exact, $\mathcal{G}_{i} \cap$ Ker $g$ is locally finitely generated. Therefore shrinking $U_{i}$ if necessary, we may assume that there is a coherent submodule $\left.\mathcal{H} \subset \mathcal{F}^{\prime}\right|_{U}$ such that $\left.\mathcal{G}_{i} \cap \operatorname{Ker} g \subset \mathcal{H}\right|_{U_{i}}$. Replacing $\mathcal{G}_{i}$ with $\mathcal{G}_{i}+\left.\mathcal{H}\right|_{U_{i}}$, we may assume from the beginning that $\mathcal{G}_{i} \cap \operatorname{Ker} g=\left.\mathcal{H}\right|_{U_{i}}$. Then we have an exact sequence

$$
\left.\left.0 \rightarrow \mathcal{H}\right|_{U_{i}} \rightarrow \mathcal{G}_{i} \rightarrow \mathcal{F}\right|_{U_{i}} \rightarrow 0 .
$$

Hence we have $\left.\mathcal{G}_{i}\right|_{U_{i} \cap U_{j}}=\left.\mathcal{G}_{j}\right|_{U_{i} \cap U_{j}}$, and there exists a coherent submodule $\mathcal{G}$ of $\left.\mathcal{F}\right|_{U}$ such that $\left.\mathcal{G}\right|_{U_{i}}=\mathcal{G}_{i}$. Then $\left.\mathcal{F}\right|_{U}=\mathcal{G}+\left.\mathcal{F}^{\prime}\right|_{U}$, and $\mathcal{F}$ is quasi-good.

Let $f: X \rightarrow Y$ be a morphism of complex manifolds. We set $d_{X / Y}=d_{X}-d_{Y}$. We denote by $\mathcal{D}_{X \rightarrow Y}$ the transfer $\left(\mathcal{D}_{X}, f^{-1} \mathcal{D}_{Y}\right)$-bimodule and we denote by $\underline{f}_{*}, \underline{f}_{\text {I }}$ and $\underline{f}^{-1}$ the functors of direct image, proper direct image and inverse image for $\mathcal{D}$-modules. For example, if $\mathcal{N} \in \mathrm{D}^{\mathrm{b}}\left(\mathcal{D}_{Y}\right)$, then $\underline{f}^{-1} \mathcal{N}=\mathcal{D}_{X \rightarrow Y} \stackrel{\mathrm{L}}{\otimes_{f^{-1}} \mathcal{D}_{Y}} f^{-1} \mathcal{N}$.

Notations 2.1.2. (a) We write for short $\otimes_{\mathcal{O}}, \otimes_{\mathcal{D}}, \mathcal{H}_{o} m_{\mathcal{O}}, \mathcal{H}_{o} m_{\mathcal{D}}$ instead of $\otimes_{\mathcal{O}_{X}}$, $\otimes_{\mathcal{D}_{X}}, \mathcal{H o m}_{\mathcal{O}_{X}}, \mathcal{H}^{\circ} m_{\mathcal{D}_{X}}$.

(b) In $\S \S 3-5$, when there is no risk of confusion, we shall not write the symbols "R" and "L" of right and left derived functors, for short.

(c) Recall that:

$$
\mathrm{R} \operatorname{Hom}(\cdot, \cdot)=\mathrm{R} \Gamma(X ; \mathrm{R} \mathcal{H} o m(\cdot, \cdot)) .
$$

2.2. Review on formal and moderate cohomology. We shall briefly recall some constructions of $[\mathrm{K}]$ and $[\mathrm{K}-\mathrm{S} 2]$. Let $X$ be a real analytic manifold. The functors:

$$
\begin{aligned}
\cdot{ }^{w} \mathcal{C}_{X}^{\infty} & : \quad \mathbb{R}-\operatorname{cons}\left(\mathbb{C}_{X}\right) \rightarrow \operatorname{Mod}\left(\mathcal{D}_{X}\right), \\
\operatorname{Thom}\left(\cdot, \mathcal{D} b_{X}\right) & : \quad \mathbb{R}-\operatorname{cons}\left(\mathbb{C}_{X}\right)^{\text {opp }} \rightarrow \operatorname{Mod}\left(\mathcal{D}_{X}\right)
\end{aligned}
$$


are characterized by the following properties:

(1) they are exact functors,

(2) if $U$ is an open subanalytic subset of $X$ and $Z=X \backslash U$, then $\mathbb{C}_{U} \stackrel{w}{\otimes} \mathcal{C}_{X}^{\infty}$ is the sheaf of $\mathcal{C}^{\infty}$-functions vanishing up to infinite order on $Z$, and $\mathcal{T h o m}\left(\mathbb{C}_{Z}, \mathcal{D} b_{X}\right)$ is the sheaf $\Gamma_{Z}\left(\mathcal{D} b_{X}\right)$ of distributions supported by $Z$.

These functors being exact, they extend naturally as functors on the derived categories:

$$
\begin{array}{rll}
\stackrel{w}{\otimes}^{\infty} \mathcal{C}_{X}^{\infty} & : \quad \mathrm{D}_{\mathbb{R}-\mathrm{c}}^{\mathrm{b}}\left(\mathbb{C}_{X}\right) \rightarrow \mathrm{D}^{\mathrm{b}}\left(\mathcal{D}_{X}\right), \\
\operatorname{Thom}\left(\cdot, \mathcal{D} b_{X}\right) & : \quad\left(\mathrm{D}_{\mathbb{R}-\mathrm{c}}^{\mathrm{b}}\left(\mathbb{C}_{X}\right)\right)^{\mathrm{opp}} \rightarrow \mathrm{D}^{\mathrm{b}}\left(\mathcal{D}_{X}\right) .
\end{array}
$$

Now let $X$ be a complex manifold, $X^{\mathbb{R}}$ the real analytic underlying manifold and $\bar{X}$ the complex conjugate manifold. If there is no risk of confusion, we write $X$ instead of $X^{\mathbb{R}}$ (e.g.: we write $\left.\mathcal{C}_{X}^{\infty}\right)$. The functors

$$
\begin{array}{rll}
\cdot \stackrel{w}{\otimes} \mathcal{O}_{X} & : & \mathrm{D}_{\mathbb{R}-\mathrm{c}}^{\mathrm{b}}\left(\mathbb{C}_{X}\right) \rightarrow \mathrm{D}^{\mathrm{b}}\left(\mathcal{D}_{X}\right), \\
\operatorname{Thom}\left(\cdot, \mathcal{O}_{X}\right) & : \quad\left(\mathrm{D}_{\mathbb{R}-\mathrm{c}}^{\mathrm{b}}\left(\mathbb{C}_{X}\right)\right)^{\mathrm{opp}} \rightarrow \mathrm{D}^{\mathrm{b}}\left(\mathcal{D}_{X}\right)
\end{array}
$$

are defined as the Dolbeault complexes of the preceding ones, that is:

$$
\begin{aligned}
F \stackrel{w}{\otimes} \mathcal{O}_{X} & =\mathrm{R} \mathcal{H} o m_{\mathcal{D}_{\bar{X}}}\left(\mathcal{O}_{\bar{X}}, F \stackrel{w}{\otimes} \mathcal{C}_{X}^{\infty}\right), \\
\operatorname{Thom}\left(F, \mathcal{O}_{X}\right) & =\mathrm{R} \mathcal{H} o m_{\mathcal{D}_{\bar{X}}}\left(\mathcal{O}_{\bar{X}}, \operatorname{Thom}\left(F, \mathcal{D} b_{X}\right)\right) .
\end{aligned}
$$

We call these functors the functors of formal and moderate cohomology, respectively. Recall that the functor of moderate cohomology was introduced in $[\mathrm{K}]$ and that of formal cohomology in [K-S2].

There are natural morphisms

(2.2.1) $F \otimes \mathcal{O}_{X} \rightarrow F \stackrel{w}{\otimes} \mathcal{O}_{X} \rightarrow \operatorname{Thom}\left(D_{X}^{\prime} F, \mathcal{O}_{X}\right) \rightarrow R \mathcal{H o m}\left(D_{X}^{\prime} F, \mathcal{O}_{X}\right)$.

Example 2.2.1. Let $M$ be a real analytic manifold, and let

$$
i: M \hookrightarrow X
$$

be a complexification of $M$. Let $F \in D_{\mathbb{R}-c}^{b}\left(\mathbb{C}_{M}\right)$. Then we have isomorphisms

$$
\begin{aligned}
i_{*} F \stackrel{w}{\otimes} \mathcal{O}_{X} & \simeq i_{*}\left(F \stackrel{w}{\otimes} \mathcal{C}_{M}^{\infty}\right), \\
\operatorname{Thom}\left(D_{X}^{\prime}\left(i_{*} F\right), \mathcal{O}_{X}\right) & \simeq i_{*} \operatorname{Thom}\left(D_{M}^{\prime}(F), \mathcal{D} b_{M}\right) .
\end{aligned}
$$

The last isomorphism is due to Andronikof [A]. In particular, we have

$$
\begin{aligned}
\mathbb{C}_{M} \stackrel{w}{\otimes} \mathcal{O}_{X} & \simeq i_{*} \mathcal{C}_{M}^{\infty}, \\
\operatorname{Thom}\left(D_{X}^{\prime}\left(\mathbb{C}_{M}\right), \mathcal{O}_{X}\right) & \simeq \mathcal{D} b_{M},
\end{aligned}
$$

and (2.2.1) gives the classical morphisms

$$
\mathcal{A}_{M} \rightarrow \mathcal{C}_{M}^{\infty} \rightarrow \mathcal{D} b_{M} \rightarrow \mathcal{B}_{M}
$$

Example 2.2.2. Let $Z$ be a closed complex analytic subset of $X$. Then there are isomorphisms:

$$
\begin{aligned}
\mathbb{C}_{Z} \stackrel{w}{\otimes} \mathcal{O}_{X} & \simeq \mathcal{O}_{X} \widehat{\mid}_{Z}, \\
\operatorname{Thom}\left(\mathbb{C}_{Z}, \mathcal{O}_{X}\right) & \simeq \mathrm{R} \Gamma_{[Z]} \mathcal{O}_{X},
\end{aligned}
$$

where $\mathcal{O}_{X} \widehat{\mid}_{Z}$ denotes the formal completion of $\mathcal{O}_{X}$ along $Z$, and $\mathrm{R} \Gamma_{[Z]} \mathcal{O}_{X}$ denotes the algebraic relative cohomology of $\mathcal{O}_{X}$ with supports in $Z$. 
Example 2.2.3. Let $U$ be a relatively compact Stein open subanalytic subset of $X$. Then $R \Gamma\left(X ; \operatorname{Thom}\left(\mathbb{C}_{U}, \mathcal{O}_{X}\right)\right)$ is concentrated in degree 0 and coincides with the subspace of $\Gamma\left(U ; \mathcal{O}_{X}\right)$ of holomorphic functions with tempered growth at the boundary.

We have a kind of multiplication of the functors of formal and moderate cohomology. For $F, G \in \mathrm{D}_{\mathbb{R}-\mathrm{c}}^{\mathrm{b}}\left(\mathbb{C}_{X}\right)$, we have functorial morphisms ([K-S2, (5.21), (5.22), Prop. 10.6]):

$$
\begin{aligned}
& \left(F \stackrel{w}{\otimes} \mathcal{O}_{X}\right) \otimes_{\mathcal{O}}\left(G \stackrel{w}{\otimes} \mathcal{O}_{X}\right) \rightarrow(F \otimes G) \stackrel{w}{\otimes} \mathcal{O}_{X}, \\
& \text { Thom }\left(F, \mathcal{O}_{X}\right) \otimes_{\mathcal{O}}\left((F \otimes G) \stackrel{w}{\otimes} \mathcal{O}_{X}\right) \rightarrow G \stackrel{w}{\otimes} \mathcal{O}_{X}, \\
& \text { Thom }\left(F, \mathcal{O}_{X}\right) \otimes_{\mathcal{O}} \operatorname{Thom}\left(G, \mathcal{O}_{X}\right) \rightarrow \operatorname{Thom}\left(F \otimes G, \mathcal{O}_{X}\right) .
\end{aligned}
$$

The functors of formal and moderate cohomology are dual to each other in the following sense. Let $\mathrm{D}^{\mathrm{b}}(F N)$ (resp. $\mathrm{D}^{\mathrm{b}}(D F N)$ ) denote the bounded derived category of the additive category of $\mathbb{C}$-vector spaces of Fréchet nuclear (resp. dual of Fréchet nuclear) type (see [K-S2] for a precise construction).

Proposition 2.2.4 ([K-S2], Prop. 5.2). Let $F \in \mathrm{D}_{\mathbb{R}-\mathrm{c}}^{\mathrm{b}}\left(\mathbb{C}_{X}\right)$. Then we can define

$$
\mathrm{R} \Gamma\left(X ; F \stackrel{w}{\otimes} \mathcal{O}_{X}\right) \text { and } \mathrm{R} \Gamma_{c}\left(X ; \operatorname{Thom}\left(F, \Omega_{X}\right)\right)\left[d_{X}\right]
$$

as objects of $\mathrm{D}^{\mathrm{b}}(F N)$ and $\mathrm{D}^{\mathrm{b}}(D F N)$ respectively, and they are dual to each other.

\section{INTEGRAL TRANSFORMS WITH EXPONENTIAL KERNELS}

In this section, if there is no risk of confusion, we shall not write the symbols $\mathrm{R}$ and $\mathrm{L}$ of right and left derived functors, for short.

3.1. Construction of morphisms. Let $Z$ be a complex manifold, $S$ a closed hypersurface of $Z$ and $\mathcal{O}_{Z}(* S)$ the sheaf of meromorphic functions on $Z$ whose poles are contained in $S$. For an $\mathcal{O}_{Z}$-module $\mathcal{F}$, set

$$
\mathcal{F}(* S)=\mathcal{O}_{Z}(* S) \otimes_{\mathcal{O}_{Z}} \mathcal{F} .
$$

Let $\varphi$ be a global section of $\mathcal{O}_{Z}(* S)$. We introduce the sets:

$$
\begin{aligned}
& A=\{x \in Z \backslash S ; \operatorname{Re} \varphi(x) \geq 0\}, \\
& U=\{x \in Z \backslash S ; \operatorname{Re} \varphi(x)>-1\} .
\end{aligned}
$$

We introduce the left $\mathcal{D}_{Z}$-modules

$$
\begin{aligned}
\mathcal{L} & =\left(\mathcal{D}_{Z} e^{\varphi}\right)(* S), \\
\mathcal{L}^{\prime} & =\left(\mathcal{D}_{Z} e^{-\varphi}\right)(* S) .
\end{aligned}
$$

More precisely, $\mathcal{D}_{Z} e^{\varphi}$ is the $\mathcal{D}_{Z}$-module $\mathcal{D}_{Z} / \mathcal{I}$ where $\mathcal{I}$ is the left coherent ideal $\left\{P \in \mathcal{D}_{Z} ; P e^{\varphi}=0\right.$ on $\left.Z \backslash S\right\}$. Hence $\mathcal{L}$ is a holonomic $\mathcal{D}_{Z}$-module which satisfies:

$$
\mathcal{L} \simeq \mathcal{L} \otimes_{\mathcal{O}} \mathcal{O}_{Z}(* S)
$$

Moreover, $\mathcal{L}$ is an invertible $\mathcal{O}_{Z}(* S)$-module and

$$
\mathcal{L}^{\prime} \simeq \mathcal{H o m}_{\mathcal{O}_{Z}(* S)}\left(\mathcal{L}, \mathcal{O}_{Z}(* S)\right)
$$

as an $\mathcal{O}_{Z}(* S)$-module. 
Lemma 3.1.1. For $G \in \mathrm{D}_{\mathbb{R}-\mathrm{c}}^{\mathrm{b}}\left(\mathbb{C}_{Z}\right)$, we have isomorphisms

$$
\begin{gathered}
\mathcal{H}_{\mathcal{O}}\left(\mathcal{L}, G_{X \backslash S} \stackrel{w}{\otimes} \mathcal{O}_{Z}\right) \stackrel{\sim}{\rightarrow} \mathcal{H} \operatorname{om}_{\mathcal{O}}\left(\mathcal{L}, G \stackrel{w}{\otimes} \mathcal{O}_{Z}\right), \\
\operatorname{Thom}\left(G, \Omega_{Z}\right) \otimes_{\mathcal{O}} \mathcal{L} \stackrel{\sim}{\rightarrow} \operatorname{Thom}\left(G_{X \backslash S}, \Omega_{Z}\right) \otimes_{\mathcal{O}} \mathcal{L} .
\end{gathered}
$$

Proof. We have the chain of isomorphisms

$$
\begin{aligned}
\mathcal{H}_{o m_{\mathcal{O}}}\left(\mathcal{L}, G \stackrel{w}{\otimes} \mathcal{O}_{Z}\right) & \simeq \mathcal{H o m}_{\mathcal{O}}\left(\mathcal{L} \otimes \mathcal{O}_{Z}(* S), G \stackrel{w}{\otimes} \mathcal{O}_{Z}\right) \\
& \simeq \mathcal{H} \operatorname{com}_{\mathcal{O}}\left(\mathcal{L}, \mathcal{H} o m_{\mathcal{O}}\left(\mathcal{O}(* S), G \stackrel{w}{\otimes} \mathcal{O}_{Z}\right)\right) \\
& \simeq \mathcal{H} \operatorname{sm}_{\mathcal{O}}\left(\mathcal{L}, G_{Z \backslash S} \stackrel{w}{\otimes} \mathcal{O}_{Z}\right),
\end{aligned}
$$

where the last isomorphism follows from a theorem of Björk (see [B] and also [K-S2, Th. 10.7]).

The second isomorphism is proved similarly.

Lemma 3.1.2. For $G \in \mathrm{D}_{\mathbb{R}-\mathrm{c}}^{\mathrm{b}}\left(\mathbb{C}_{Z}\right)$, there are natural $\mathcal{D}_{Z}$-linear morphisms:

$$
\begin{aligned}
& \mathcal{L}^{\prime} \rightarrow \operatorname{Thom}\left(\mathbb{C}_{U}, \mathcal{O}_{Z}\right), \\
& \mathcal{H} \operatorname{om}_{\mathcal{O}}\left(\mathcal{L}, G \stackrel{w}{\otimes} \mathcal{O}_{Z}\right) \rightarrow G_{A} \stackrel{w}{\otimes} \mathcal{O}_{Z}, \\
& \operatorname{Thom}\left(G_{A}, \mathcal{O}_{Z}\right) \rightarrow \mathcal{L} \otimes_{\mathcal{O}} \operatorname{Thom}\left(G, \mathcal{O}_{Z}\right) .
\end{aligned}
$$

Proof. (i) Since $\operatorname{Re}(-\varphi)$ is bounded on $U$, the holomorphic function $e^{-\varphi}$ defines a section of the sheaf Thom $\left(\mathbb{C}_{U}, \mathcal{O}_{Z}\right)$. Hence it induces a $\mathcal{D}_{Z}$-linear morphism $\mathcal{D}_{Z} e^{-\varphi} \rightarrow \operatorname{Thom}\left(\mathbb{C}_{U}, \mathcal{O}_{Z}\right)$. Since $U \cap S=\emptyset$, this morphism factorizes through $\mathcal{L}^{\prime}$.

(ii) Since $G_{Z \backslash S} \stackrel{w}{\otimes} \mathcal{O}_{Z}$ is an $\mathcal{O}_{Z}(* S)$-module, we have:

$$
\begin{aligned}
\mathcal{H}_{o}\left(\mathcal{O}, G_{Z \backslash S} \stackrel{w}{\otimes} \mathcal{O}_{Z}\right) & \simeq \mathcal{H} \operatorname{mom}_{\mathcal{O}_{Z}(* S)}\left(\mathcal{L}, G_{Z \backslash S} \stackrel{w}{\otimes} \mathcal{O}_{Z}\right) \\
& \simeq \mathcal{L}^{\prime} \otimes_{\mathcal{O}_{Z}(* S)}\left(G_{Z \backslash S} \stackrel{w}{\otimes} \mathcal{O}_{Z}\right) \\
& \simeq \mathcal{L}^{\prime} \otimes_{\mathcal{O}}\left(G_{Z \backslash S} \stackrel{w}{\otimes} \mathcal{O}_{Z}\right) .
\end{aligned}
$$

Similarly, one has:

$$
\mathcal{L} \otimes_{\mathcal{O}} \operatorname{Thom}\left(G_{Z \backslash S}, \mathcal{O}_{Z}\right) \simeq \mathcal{H} \operatorname{Tom}_{\mathcal{O}}\left(\mathcal{L}^{\prime}, \operatorname{Thom}\left(G_{Z \backslash S}, \mathcal{O}_{Z}\right)\right) .
$$

(iii) Let us construct the morphism (3.1.4). Since $A$ is closed in $Z \backslash S$, we have the morphism:

$$
G_{Z \backslash S} \stackrel{w}{\otimes} \mathcal{O}_{Z} \rightarrow G_{A} \stackrel{w}{\otimes} \mathcal{O}_{Z}
$$

Applying Thom $\left(\mathbb{C}_{U}, \mathcal{O}_{Z}\right) \otimes_{\mathcal{O}} \cdot$ and using (3.1.3) we get:

$$
\mathcal{L}^{\prime} \otimes_{\mathcal{O}}\left(G_{Z \backslash S} \stackrel{w}{\otimes} \mathcal{O}_{Z}\right) \rightarrow \operatorname{Thom}\left(\mathbb{C}_{U}, \mathcal{O}_{Z}\right) \otimes_{\mathcal{O}}\left(G_{A} \stackrel{w}{\otimes} \mathcal{O}_{Z}\right)
$$

Since $A=A \cap U$, we have $\mathbb{C}_{A} \simeq \mathbb{C}_{U} \otimes \mathbb{C}_{A}$, and the morphism (2.2.3) sends the right hand side of (3.1.6) to $G_{A} \stackrel{w}{\otimes} \mathcal{O}_{Z}$. Then the result follows from Lemma 3.1.1 and (ii).

(iv) Let us construct the morphism (3.1.5). By the results of Lemma 3.1.1 and (ii), it is enough to construct

$$
\mathcal{L}^{\prime} \otimes_{\mathcal{O}} \operatorname{Thom}\left(G_{A}, \mathcal{O}_{Z}\right) \rightarrow \operatorname{Thom}\left(G_{Z \backslash S}, \mathcal{O}_{Z}\right) .
$$

This last morphism is deduced from (3.1.3) and (2.2.4). 
In the sequel, we shall have to consider two meromorphic functions $\varphi_{1}$ and $\varphi_{2}$ with poles in $S$. We set:

and we define for $j=0,1,2$ :

$$
\varphi_{0}=\varphi_{1}+\varphi_{2}
$$

$$
\begin{aligned}
& A_{j}=\left\{x \in Z \backslash S ; \operatorname{Re} \varphi_{j} \geq 0\right\}, \\
& \mathcal{L}_{j}=\left(\mathcal{D}_{Z} e^{\varphi_{j}}\right)(* S) .
\end{aligned}
$$

Lemma 3.1.3. (i) We have:

$$
\mathcal{L}_{1} \otimes_{\mathcal{O}} \mathcal{L}_{2} \simeq \mathcal{L}_{0}
$$

(ii) The diagram below commutes:

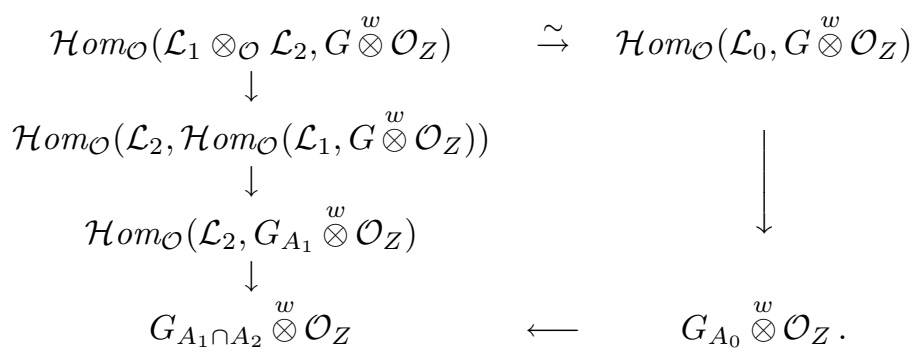

Here, the horizontal arrow in the bottom row is induced by $\mathbb{C}_{A_{0}} \rightarrow \mathbb{C}_{A_{1} \cap A_{2}}$.

There is a similar result with $\operatorname{Thom}(\cdot, \mathcal{O})$.

Proof. The proof is straightforward.

Now consider a correspondence of complex manifolds

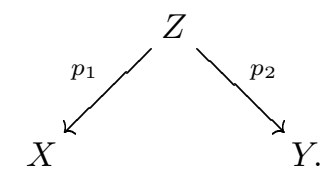

We shall assume:

$$
p_{1} \text { and } p_{2} \text { are proper. }
$$

(This hypothesis could be weakened, see $[\mathrm{K}-\mathrm{S} 2, \S 7]$ and $\S 4$.)

Let $F \in \mathrm{D}_{\mathbb{R}-\mathrm{c}}^{\mathrm{b}}\left(\mathbb{C}_{X}\right), K \in \mathrm{D}_{\mathbb{R}-\mathrm{c}}^{\mathrm{b}}\left(\mathbb{C}_{Z}\right), \mathcal{N} \in \mathrm{D}_{\text {q-good }}^{\mathrm{b}}\left(\mathcal{D}_{Y}\right)$ and $\mathcal{K} \in \mathrm{D}_{\mathrm{q}-\text { good }}^{\mathrm{b}}\left(\mathcal{D}_{Z}\right)$. We set:

$$
\left\{\begin{array}{l}
\mathcal{K} \circ \mathcal{N}=\underline{p}_{1 *}\left(\mathcal{K} \otimes \mathcal{O} \underline{p}_{2}^{-1} \mathcal{N}\right) \\
F \circ K=p_{2 !}\left(p_{1}^{-1} F \otimes K\right)
\end{array}\right.
$$

Assume for a while that $\mathcal{K}$ is regular holonomic and that $K=\operatorname{Sol}(\mathcal{K})$ (hence $\mathcal{K}=\operatorname{Thom}\left(K, \mathcal{O}_{Z}\right)$ by $\left.[\mathrm{K}]\right)$. We have the chain of isomorphisms:

$$
\begin{aligned}
\operatorname{Hom}_{\mathcal{D}}\left(\mathcal{K} \circ \mathcal{N}, F \stackrel{w}{\otimes} \mathcal{O}_{X}\right)\left[d_{X}\right] & \simeq \operatorname{Hom}_{\mathcal{D}}\left(\mathcal{K} \otimes \mathcal{O} \underline{p}_{2}^{-1} \mathcal{N}, p_{1}^{-1} F \stackrel{w}{\otimes} \mathcal{O}_{Z}\right)\left[d_{Z}\right] \\
& \simeq \operatorname{Hom}_{\mathcal{D}}\left(\underline{p}_{2}^{-1} \mathcal{N}, \mathcal{H} \circ m_{\mathcal{O}}\left(\mathcal{K}, p_{1}^{-1} F \stackrel{w}{\otimes} \mathcal{O}_{Z}\right)\right)\left[d_{Z}\right] \\
& \simeq \operatorname{Hom}_{\mathcal{D}}\left(\underline{p}_{2}^{-1} \mathcal{N},\left(p_{1}^{-1} F \otimes K\right) \stackrel{w}{\otimes} \mathcal{O}_{Z}\right)\left[d_{Z}\right] \\
& \simeq \operatorname{Hom}_{\mathcal{D}}\left(\mathcal{N},(F \circ K) \stackrel{w}{\otimes} \mathcal{O}_{Y}\right)\left[d_{Z}\right] .
\end{aligned}
$$


The first isomorphism is obtained in [K-S2, Th. 7.3], the third one in [K-S2, Th. 10.7], and the fourth one in [K-S2, Th. 7.2].

Similarly, we have the chain of isomorphisms:

$$
\begin{aligned}
\Gamma(Y ; \operatorname{Thom} & \left.\left(F \circ K, \Omega_{Y}\right) \otimes_{\mathcal{D}} \mathcal{N}\right)\left[d_{Y}\right] \simeq \Gamma\left(Z ; \operatorname{Thom}\left(p_{1}^{-1} F \otimes K, \Omega_{Z}\right) \otimes_{\mathcal{D}} \underline{p}_{2}^{-1} \mathcal{N}\right)\left[d_{Z}\right] \\
& \simeq \Gamma\left(Z ;\left(\operatorname{Thom}\left(p_{1}^{-1} F, \Omega_{Z}\right) \otimes_{\mathcal{O}} \mathcal{K}\right) \otimes_{\mathcal{D}} \underline{p}_{2}^{-1} \mathcal{N}\right)\left[d_{Z}\right] \\
& \simeq \Gamma\left(Z ; \operatorname{Thom}\left(p_{1}^{-1} F, \Omega_{Z}\right) \otimes_{\mathcal{D}}\left(\mathcal{K} \otimes_{\mathcal{O}} \underline{p}_{2}^{-1} \mathcal{N}\right)\right)\left[d_{Z}\right] \\
& \simeq \Gamma\left(X ; \operatorname{Thom}\left(F, \Omega_{X}\right) \otimes_{\mathcal{D}}(\mathcal{K} \circ \mathcal{N})\right)\left[d_{Z}\right]
\end{aligned}
$$

Summarizing, we have constructed the isomorphisms:

$$
\begin{aligned}
\Gamma\left(X ; \mathcal{H o m}_{\mathcal{D}}\left(\mathcal{K} \circ \mathcal{N}, F \stackrel{w}{\otimes} \mathcal{O}_{X}\right)\right) \stackrel{\sim}{\rightarrow} \Gamma\left(Y ; \mathcal{H} \operatorname{mom}_{\mathcal{D}}\left(\mathcal{N},(F \circ K) \stackrel{w}{\otimes} \mathcal{O}_{Y}\right)\right)\left[d_{Z / X}\right], \\
\Gamma\left(Y ; \operatorname{Thom}\left(F \circ K, \Omega_{Y}\right) \otimes_{\mathcal{D}} \mathcal{N}\right) \stackrel{\stackrel{\sim}{\rightarrow}}{ } \Gamma\left(X ; \operatorname{Thom}\left(F, \Omega_{X}\right) \otimes_{\mathcal{D}}(\mathcal{K} \circ \mathcal{N})\right)\left[d_{Z / Y}\right]
\end{aligned}
$$

(see [K-S2, Th. 10.8]).

Next we consider the case of irregular kernels. Let $\varphi$ be a meromorphic function on $Z$ with poles in a closed hypersurface $S$ of $Z$, and set as above:

$$
\begin{aligned}
& A=\{x \in Z \backslash S ; \operatorname{Re} \varphi(x) \geq 0\}, \\
& \mathcal{L}=\left(\mathcal{D}_{Z} e^{\varphi}\right)(* S) .
\end{aligned}
$$

In the construction of the isomorphism (3.1.9), if we take $\mathcal{L}$ as $\mathcal{K}$, the isomorphism

$$
\mathcal{H}_{o m_{\mathcal{O}}}\left(\mathcal{K}, p_{1}^{-1} F \stackrel{w}{\otimes} \mathcal{O}_{Z}\right) \simeq\left(p_{1}^{-1} F \otimes K\right) \stackrel{w}{\otimes} \mathcal{O}_{Z}
$$

does not hold any more, but we may replace it by the morphism (3.1.4):

$$
\mathcal{H o m}_{\mathcal{O}}\left(\mathcal{L}, p_{1}^{-1} F \stackrel{w}{\otimes} \mathcal{O}_{Z}\right) \rightarrow\left(p_{1}^{-1} F \otimes \mathbb{C}_{A}\right) \stackrel{w}{\otimes} \mathcal{O}_{Z}
$$

Hence, we get the morphism $L_{\varphi}$ :

$$
\begin{aligned}
\Gamma\left(X ; \mathcal{H o m}_{\mathcal{D}}\left(\mathcal{L} \circ \mathcal{N}, F \stackrel{w}{\otimes} \mathcal{O}_{X}\right)\right) \\
\underset{L_{\varphi}}{\longrightarrow} \Gamma\left(Y ; \mathcal{H} o m_{\mathcal{D}}\left(\mathcal{N},\left(F \circ \mathbb{C}_{A}\right) \stackrel{w}{\otimes} \mathcal{O}_{Y}\right)\right)\left[d_{Z / X}\right] .
\end{aligned}
$$

Similarly, using (3.1.5), we get the morphism ${ }^{t} L_{\varphi}$ :

$$
\begin{aligned}
\Gamma\left(Y ; \operatorname{Thom}\left(F \circ \mathbb{C}_{A}, \Omega_{Y}\right) \otimes_{\mathcal{D}} \mathcal{N}\right) \\
\underset{{ }^{t} L_{\varphi}}{\longrightarrow} \Gamma\left(X ; \operatorname{Thom}\left(F, \Omega_{X}\right) \otimes_{\mathcal{D}}(\mathcal{L} \circ \mathcal{N})\right)\left[d_{Z / Y}\right] .
\end{aligned}
$$

3.2. Comparison with regular kernels. We shall have to compare the morphisms (3.1.11) and (3.1.12) with the adjunction morphisms associated to regular holonomic kernels.

Let $\mathcal{L}, \varphi, A$ be as above and let $\mathcal{K}$ be a regular holonomic $\mathcal{D}_{Z}$-module, $K=$ $\operatorname{Sol}(\mathcal{K})$. We assume to be given a $\mathcal{D}_{Z}$-linear morphism:

$$
\mathcal{K} \rightarrow \mathcal{L} .
$$

This morphism defines morphisms:

$$
\mathbb{C}_{X \backslash S} \simeq \operatorname{Sol}(\mathcal{L})_{X \backslash S} \rightarrow K_{X \backslash S} \rightarrow K .
$$


We shall assume:

The morphism $\mathbb{C}_{X \backslash S} \rightarrow K$ factorizes as:

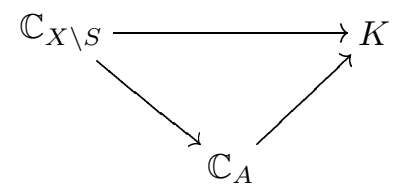

$\mathcal{K} \otimes_{\mathcal{O}} \mathcal{L}^{\prime}$ is regular holonomic.

Proposition 3.2.1. Let $\mathcal{N} \in \mathrm{D}_{\mathrm{q}-\text { good }}^{\mathrm{b}}\left(\mathcal{D}_{Y}\right), F \in \mathrm{D}_{\mathbb{R}-\mathrm{c}}^{\mathrm{b}}\left(\mathbb{C}_{X}\right)$ and consider a morphism (3.2.1). Assume (3.2.2) and (3.2.3). Then the diagram below commutes:

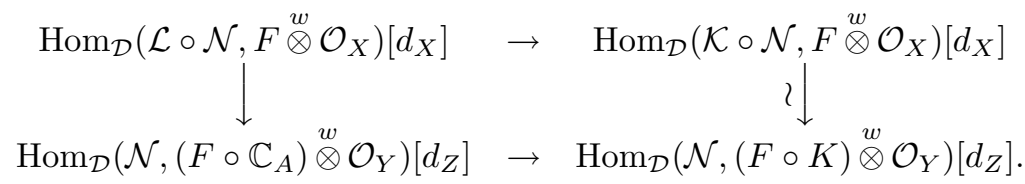

There is a similar result for Thom.

Proof. Set $G=p_{1}^{-1} F \in \mathrm{D}_{\mathbb{R}-\mathrm{c}}^{\mathrm{b}}\left(\mathbb{C}_{Z}\right)$. We can reduce the proposition to the commutativity of the diagram

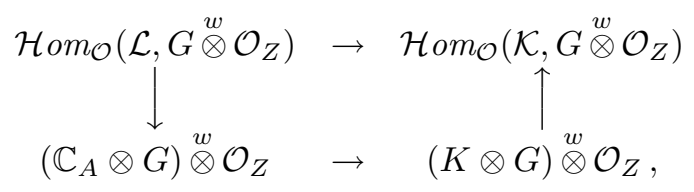

or equivalently the commutativity of

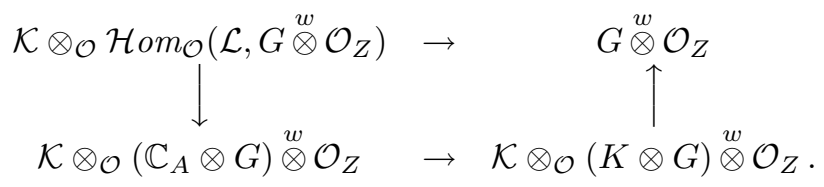

Setting $U$ as in (3.1.2), consider the diagram:

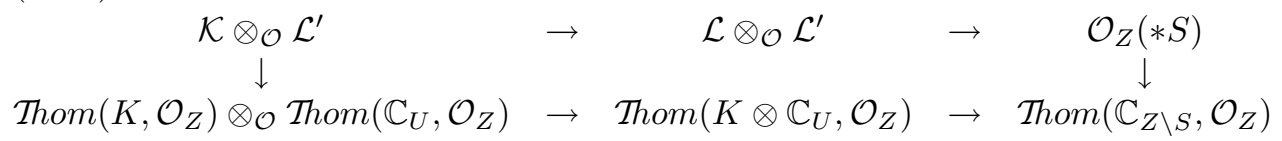

It obviously commutes on $Z \backslash S$. On the other hand, we have

$$
\begin{aligned}
& \operatorname{Hom}_{\mathrm{D}^{\mathrm{b}}\left(\mathcal{D}_{Z}\right)}\left(\mathcal{K} \otimes_{\mathcal{O}} \mathcal{L}^{\prime}, \text { Thom }\left(\mathbb{C}_{Z \backslash S}, \mathcal{O}_{Z}\right)\right) \\
& \quad \simeq \operatorname{Hom}_{\mathrm{D}^{\mathrm{b}}\left(\mathcal{D}_{Z}\right)}\left(\mathcal{K} \otimes_{\mathcal{O}} \mathcal{L}^{\prime}, \mathcal{H} \text { om }\left(\mathbb{C}_{Z \backslash S}, \mathcal{O}_{Z}\right)\right) \\
& \simeq \operatorname{Hom}_{\mathrm{D}^{\mathrm{b}}\left(\mathcal{D}_{Z \backslash S}\right)}\left(\left.\mathcal{K} \otimes_{\mathcal{O}} \mathcal{L}^{\prime}\right|_{Z \backslash S}, \mathcal{H o m}\left(\mathbb{C}_{Z \backslash S}, \mathcal{O}_{Z \backslash S}\right)\right) \\
& \simeq \operatorname{Hom}_{\mathrm{D}^{\mathrm{b}}\left(\mathcal{D}_{Z \backslash S}\right)}\left(\left.\mathcal{K} \otimes_{\mathcal{O}} \mathcal{L}^{\prime}\right|_{Z \backslash S}, \text { Thom }\left(\mathbb{C}_{Z \backslash S}, \mathcal{O}_{Z \backslash S}\right)\right) .
\end{aligned}
$$

Here the first isomorphism follows from the regularity of $\mathcal{K} \otimes_{\mathcal{O}} \mathcal{L}^{\prime}$ by $[\mathrm{K}]$. Hence we obtain the commutativity of (3.2.5). 
From (3.2.5) we deduce the commutative diagram:

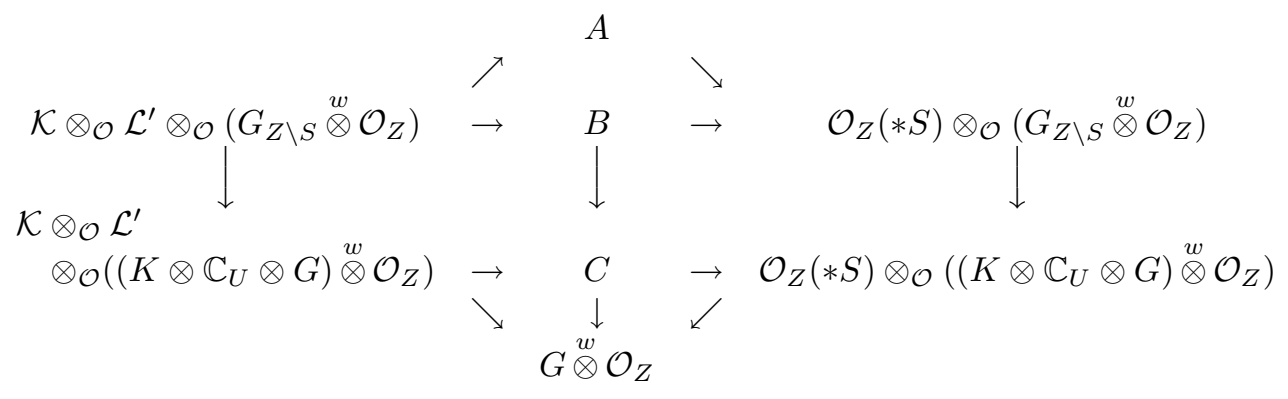

where

$$
\begin{aligned}
A & =\mathcal{L} \otimes \mathcal{O} \mathcal{L}^{\prime} \otimes \mathcal{O} \\
B & =\operatorname{Thom}\left(K \otimes G_{Z \backslash S} \stackrel{w}{\otimes} \mathcal{O}_{U}, \mathcal{O}_{Z}\right) \otimes_{\mathcal{O}}\left(G_{Z \backslash S} \stackrel{w}{\otimes} \mathcal{O}_{Z}\right), \\
C & =\operatorname{Thom}\left(K \otimes \mathbb{C}_{U}, \mathcal{O}_{Z}\right) \otimes_{\mathcal{O}}\left(\left(K \otimes \mathbb{C}_{U} \otimes G\right) \stackrel{w}{\otimes} \mathcal{O}_{Z}\right) .
\end{aligned}
$$

Hence we get the commutative diagram:

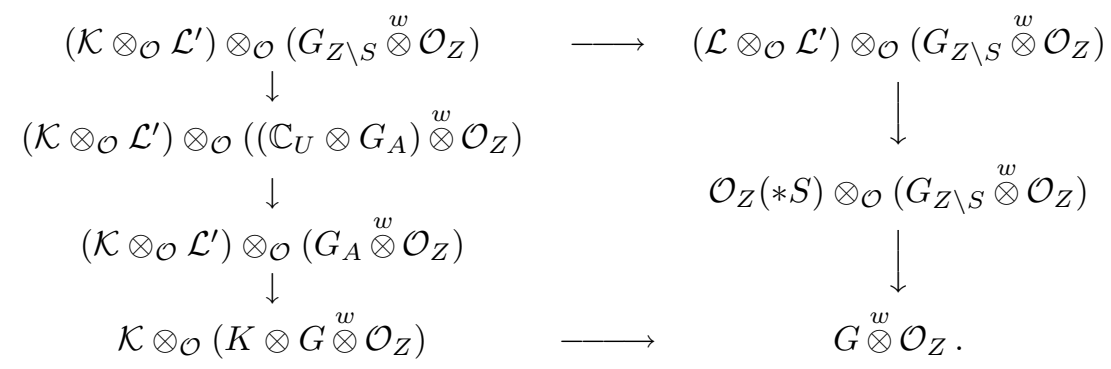

This implies the commutativity of (3.2.4).

3.3. The inversion formula. In this section we shall compose integral transforms in order to obtain an inversion formula. For the sake of brevity, we concentrate our study on the functor $\stackrel{w}{\otimes} \mathcal{O}$, leaving the details for $\operatorname{Thom}(\cdot, \mathcal{O})$ to the reader. We consider three compact complex manifolds $X, Y, Z$ and the projections:

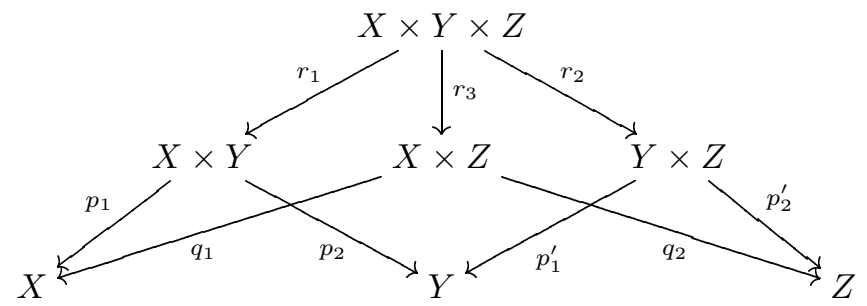

(One shall take care that the notations are not the same as in $\S \S 3.1-3.2$.

Let $\varphi_{1}$ (resp. $\varphi_{2}$ ) be a meromorphic function on $X \times Y$ (resp. $Y \times Z$ ) with poles in $S_{1}$ (resp. $S_{2}$ ) and define the meromorphic function $\varphi_{0}$ on $X \times Y \times Z$ as:

$$
\varphi_{0}(x, y, z)=\varphi_{1}(x, y)+\varphi_{2}(y, z) .
$$


Then $S_{0}:=r_{1}^{-1}\left(S_{1}\right) \cup r_{2}^{-1}\left(S_{2}\right)$ contains its poles. We also consider the sets:

$$
\begin{aligned}
& A_{1}=\left\{(x, y) \in X \times Y \backslash S_{1} ; \operatorname{Re} \varphi_{1} \geq 0\right\}, \\
& A_{2}=\left\{(y, z) \in Y \times Z \backslash S_{2} ; \operatorname{Re} \varphi_{2} \geq 0\right\}, \\
& A_{0}=\left\{(x, y, z) \in X \times Y \times Z \backslash S_{0} ; \operatorname{Re} \varphi_{0} \geq 0\right\}, \\
& A_{3}=r_{1}^{-1} A_{1} \cap r_{2}^{-1} A_{2} \subset A_{0},
\end{aligned}
$$

and the $\mathcal{D}$-modules:

$$
\begin{aligned}
\mathcal{L}_{1} & =\mathcal{D}_{X \times Y} e^{\varphi_{1}}\left(* S_{1}\right), \\
\mathcal{L}_{2} & =\mathcal{D}_{Y \times Z} e^{\varphi_{2}}\left(* S_{2}\right), \\
\mathcal{L}_{0} & =\mathcal{D}_{X \times Y \times Z} e^{\varphi_{0}}\left(* S_{0}\right) \\
& \simeq \underline{r}_{1}^{-1} \mathcal{L}_{1} \otimes \mathcal{O} \underline{r}_{2}^{-1} \mathcal{L}_{2}, \\
\mathcal{L}_{0}^{\prime} & =\mathcal{D}_{X \times Y \times Z} e^{-\varphi_{0}}\left(* S_{0}\right) .
\end{aligned}
$$

We define:

$$
\begin{aligned}
& \mathcal{L}_{1} \circ \mathcal{L}_{2}=\underline{r}_{3 *}\left(\underline{r}_{1}^{-1} \mathcal{L}_{1} \otimes \mathcal{O} \underline{r}_{2}^{-1} \mathcal{L}_{2}\right) \simeq \underline{r}_{3 *} \mathcal{L}_{0} \in \mathrm{D}^{\mathrm{b}}\left(\mathcal{D}_{X \times Z}\right), \\
& \mathbb{C}_{A_{1}} \circ \mathbb{C}_{A_{2}}=r_{3 *}\left(r_{1}^{-1} \mathbb{C}_{A_{1}} \otimes r_{2}^{-1} \mathbb{C}_{A_{2}}\right) \simeq r_{3 *} \mathbb{C}_{A_{3}} \in \mathrm{D}_{\mathbb{R}-\mathrm{c}}^{\mathrm{b}}\left(\mathbb{C}_{X \times Z}\right) .
\end{aligned}
$$

Let $\mathcal{N} \in \mathrm{D}_{\text {q-good }}^{\mathrm{b}}\left(\mathcal{D}_{Z}\right)$. One has:

$$
\begin{aligned}
\mathcal{L}_{1} \circ\left(\mathcal{L}_{2} \circ \mathcal{N}\right) & =\underline{p}_{1 *}\left(\mathcal{L}_{1} \otimes_{\mathcal{O}} \underline{p}_{2}^{-1} \underline{p}_{1 *}^{\prime}\left(\mathcal{L}_{2} \otimes \mathcal{O} \underline{p}_{2}^{\prime-1} \mathcal{N}\right)\right) \\
& \simeq \underline{p}_{1 *}\left(\mathcal{L}_{1} \otimes_{\mathcal{O}} \underline{r}_{1 *} \underline{r}_{2}^{-1}\left(\mathcal{L}_{2} \otimes \mathcal{O} \underline{p}_{2}^{\prime-1} \mathcal{N}\right)\right) \\
& \simeq \underline{p}_{1 *} \underline{r}_{1 *}\left(\left(\underline{r}_{1}^{-1} \mathcal{L}_{1} \otimes \mathcal{O} \underline{r}_{2}^{-1} \mathcal{L}_{2}\right) \otimes \mathcal{O} \underline{r}_{2}^{-1} \underline{p}_{2}^{\prime-1} \mathcal{N}\right) \\
& \simeq \underline{q}_{1 *} \underline{r}_{3 *}\left(\left(\underline{r}_{1}^{-1} \mathcal{L}_{1} \otimes \mathcal{O} \underline{r}_{2}^{-1} \mathcal{L}_{2}\right) \otimes \mathcal{O} \underline{r}_{3}^{-1} \underline{q}_{2}^{-1} \mathcal{N}\right) \\
& \simeq\left(\mathcal{L}_{1} \circ \mathcal{L}_{2}\right) \circ \mathcal{N} \\
& \simeq \mathcal{L}_{0} \circ \mathcal{N} .
\end{aligned}
$$

Here, we have used the isomorphisms:

$$
\begin{aligned}
& \underline{p}_{2}^{-1} \circ \underline{p}_{1 *}^{\prime} \simeq \underline{r}_{1 *} \circ \underline{r}_{2}^{-1}, \\
& \mathcal{L}_{1} \otimes_{\mathcal{O}}\left(\underline{r}_{1 *} \mathcal{M}_{1}\right) \simeq \underline{r}_{1 *}\left(\underline{r}_{1}^{-1} \mathcal{L}_{1} \otimes_{\mathcal{O}} \mathcal{M}_{1}\right), \\
& \underline{r}_{2}^{-1}\left(\mathcal{L}_{2} \otimes_{\mathcal{O}} \mathcal{M}_{2}\right) \simeq \underline{r}_{2}^{-1} \mathcal{L}_{2} \otimes_{\mathcal{O}} \underline{r}_{2}^{-1} \mathcal{M}_{2} .
\end{aligned}
$$

Similarly, for $F \in \mathrm{D}_{\mathbb{R}-\mathrm{c}}^{\mathrm{b}}\left(\mathcal{D}_{X}\right)$, one has:

$$
\begin{aligned}
\left(F \circ \mathbb{C}_{A_{1}}\right) \circ \mathbb{C}_{A_{2}} & =p_{2 !}^{\prime}\left(p_{1}^{-1} p_{2 !}\left(p_{1}^{-1} F \otimes \mathbb{C}_{A_{1}}\right) \otimes \mathbb{C}_{A_{2}}\right) \\
& \simeq p_{2 !}^{\prime}\left(r_{2 !} r_{1}^{-1}\left(p_{1}^{-1} F \otimes \mathbb{C}_{A_{1}}\right) \otimes \mathbb{C}_{A_{2}}\right) \\
& \simeq p_{2 !}^{\prime} r_{2 !}\left(r_{1}^{-1} p_{1}^{-1} F \otimes \mathbb{C}_{r_{1}^{-1} A_{1}} \otimes \mathbb{C}_{r_{2}^{-1} A_{2}}\right) \\
& \simeq q_{2 !} r_{3 !}\left(r_{3}^{-1} q_{1}^{-1} F \otimes \mathbb{C}_{A_{3}}\right) \\
& \simeq F \circ\left(\mathbb{C}_{A_{1}} \circ \mathbb{C}_{A_{2}}\right) \\
& \simeq F \circ \mathbb{C}_{A_{3}} .
\end{aligned}
$$


Lemma 3.3.1. The diagram below commutes:

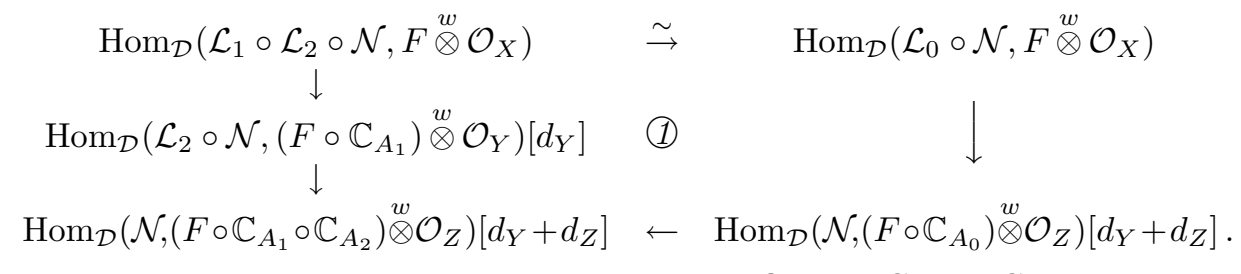

Here the horizontal arrow in the bottom row is defined by $\mathbb{C}_{A_{0}} \rightarrow \mathbb{C}_{A_{3}}$.

Proof. It follows easily from Lemma 3.1.3.

Now assume a regular holonomic $\mathcal{D}_{X \times Z}$-module $\mathcal{K}$ is given, together with a morphism:

$$
\underline{r}_{3}^{-1} \mathcal{K}\left[-d_{Y}\right] \rightarrow \mathcal{L}_{0}
$$

Let $K=\operatorname{Sol}(\mathcal{K})$. By applying the functor $S o l$ to (3.3.7), we get a morphism:

$$
\mathbb{C}_{X \times Y \times Z \backslash S_{0}} \rightarrow r_{3}^{-1} K\left[d_{Y}\right] .
$$

We shall assume that there are morphisms $\alpha: \mathbb{C}_{A_{0}} \rightarrow r_{3}^{-1} K\left[d_{Y}\right]$ and $\beta: r_{3}^{-1} K\left[d_{Y}\right]$ $\rightarrow \mathbb{C}_{A_{3}}$ such that the following diagram commutes:

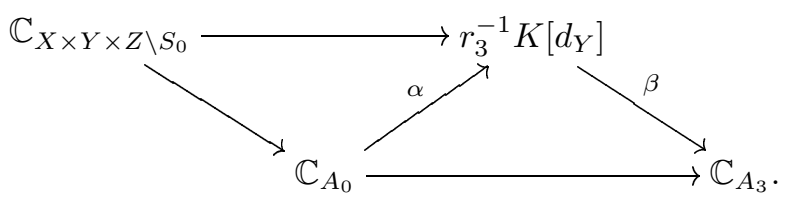

Using the morphism $r_{3 !} r_{3}^{!} K \rightarrow K$, we get the commutative diagram:

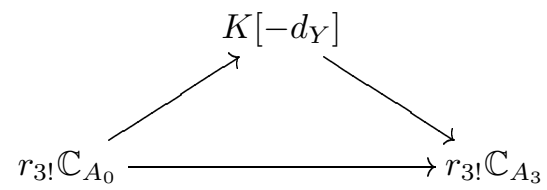

Finally, we shall assume:

$$
\underline{r}_{3}^{-1} \mathcal{K} \otimes_{\mathcal{O}} \mathcal{L}_{0}^{\prime} \quad \text { is regular holonomic. }
$$

Then Proposition 3.2.1 gives a commutative diagram:

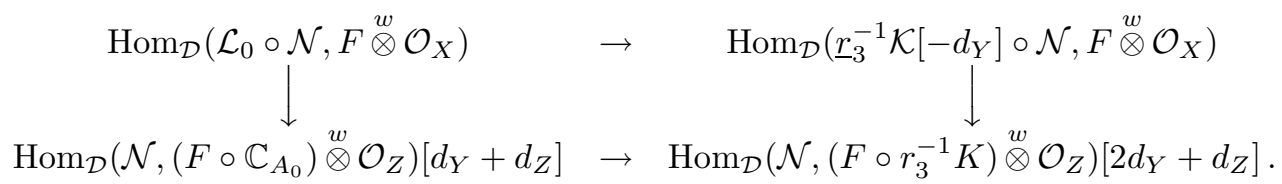

Using the natural morphisms

$$
\begin{aligned}
& \mathcal{K} \rightarrow \underline{r}_{3 *} \underline{r}_{3}^{-1} \mathcal{K}\left[-d_{Y}\right], \\
& r_{3 !} r_{3}^{-1} K\left[2 d_{Y}\right] \simeq r_{3 !} r_{3}^{!} K \rightarrow K,
\end{aligned}
$$

and noticing that

$$
\underline{r}_{3}^{-1} \mathcal{K} \circ \mathcal{N} \simeq \underline{r}_{3 *} \underline{r}_{3}^{-1} \mathcal{K} \circ \mathcal{N}
$$

and

$$
F \circ r_{3}^{-1} K \simeq F \circ\left(r_{3 !} r_{3}^{-1} K\right)
$$


we get the commutative diagram

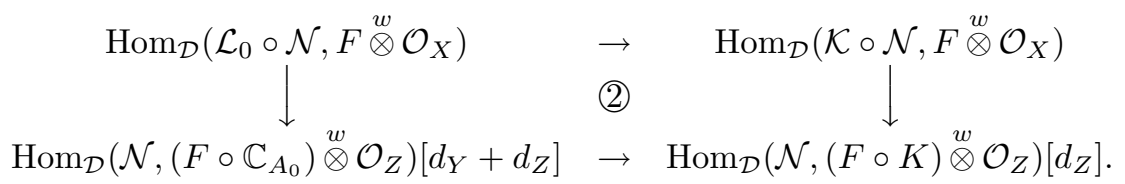

Moreover, (3.3.9) induces the commutative diagram:

$$
\begin{aligned}
\operatorname{Hom}_{\mathcal{D}}\left(\mathcal{N},\left(F \circ \mathbb{C}_{A_{0}}\right) \stackrel{w}{\otimes} \mathcal{O}_{Z}\right)\left[d_{Y}+d_{Z}\right] & \rightarrow \operatorname{Hom}_{\mathcal{D}}\left(\mathcal{N},(F \circ K) \stackrel{w}{\otimes} \mathcal{O}_{Z}\right)\left[d_{Z}\right] \\
& \downarrow \\
& \operatorname{Hom}_{\mathcal{D}}\left(\mathcal{N},\left(F \circ \mathbb{C}_{A_{1}} \circ \mathbb{C}_{A_{2}}\right) \stackrel{w}{\otimes} \mathcal{O}_{Z}\right)\left[d_{Y}+d_{Z}\right] .
\end{aligned}
$$

By putting together the diagrams (1)-(3), we obtain:

Theorem 3.3.2. Let $X, Y$ and $Z$ be compact complex manifolds, $F \in \mathrm{D}_{\mathbb{R}-\mathrm{c}}^{\mathrm{b}}\left(\mathbb{C}_{X}\right)$, and $\mathcal{N} \in \mathrm{D}_{\mathrm{q} \text {-good }}^{\mathrm{b}}\left(\mathcal{D}_{Z}\right)$. Assume a morphism $(3.3 .7)$ is given with $\mathcal{K}$ regular holonomic satisfying (3.3.8) and (3.3.10). Then the diagram below commutes:

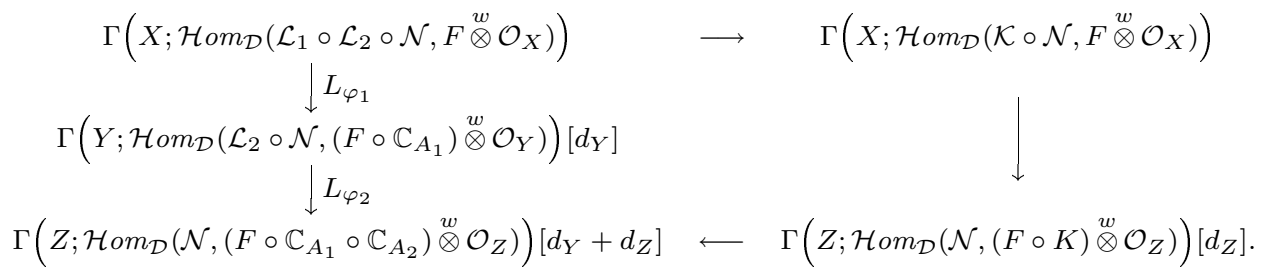

Using the same hypotheses, one similarly obtains a commutative diagram:

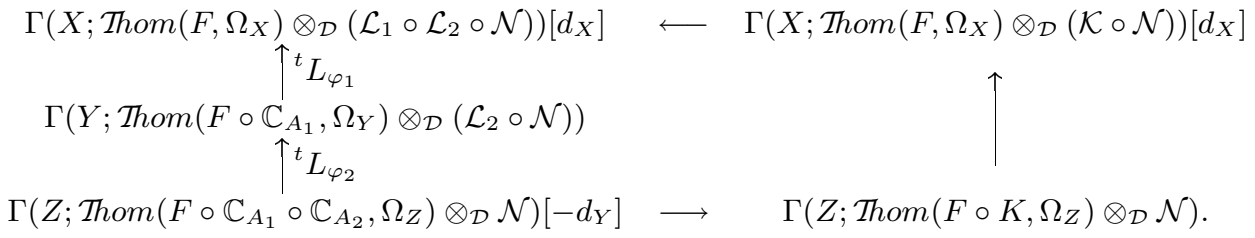

Corollary 3.3.3. Assume $X=Z, F \circ \mathbb{C}_{A_{2}} \circ \mathbb{C}_{A_{1}} \simeq F, \mathcal{L}_{1} \circ \mathcal{L}_{2} \circ \mathcal{N} \simeq \mathcal{N}, F \circ K \simeq F$ and $\mathcal{K} \circ \mathcal{N} \simeq \mathcal{N}$. Then $L_{\varphi_{2}} \circ L_{\varphi_{1}}=\mathrm{id}$.

If the same result holds with $\varphi_{1}$ and $\varphi_{2}$ interchanged, we get that $L_{\varphi_{1}}$ and $L_{\varphi_{2}}$ are inverse to each other. That is the reason why we may consider Theorem 3.3.2 as an "inversion formula".

\section{Algebraic Setting}

In this section, if there is no risk of confusion, we shall not write the symbols $\mathrm{R}$ and $\mathrm{L}$ of right and left derived functors, for short.

4.1. Definitions. In the sequel, we work on complex algebraic varieties (i.e. separated schemes of finite type over $\mathbb{C}$ ). For a complex algebraic variety $X$, we denote by $\mathcal{O}_{X}$ the structural sheaf, and by $\mathcal{D}_{X}$ the sheaf of differential operators. We say that a $\mathcal{D}_{X}$-module is quasi-good, if it is quasi-coherent as an $\mathcal{O}_{X}$-module and locally generated by countably many sections. We denote by $\mathrm{D}_{\mathrm{q} \text {-good }}^{\mathrm{b}}\left(\mathcal{D}_{X}\right)$ the full subcategory of $\mathrm{D}^{\mathrm{b}}\left(\mathcal{D}_{X}\right)$ consisting of objects whose cohomology groups are quasi-good. Then $\cdot \otimes_{\mathcal{O}_{X}} \cdot$ gives a functor

$$
\cdot \otimes_{\mathcal{O}} \cdot \quad: \quad \mathrm{D}_{\mathrm{q}-\text { good }}^{\mathrm{b}}\left(\mathcal{D}_{X}\right) \times \mathrm{D}_{\mathrm{q}-\text { good }}^{\mathrm{b}}\left(\mathcal{D}_{X}\right) \rightarrow \mathrm{D}_{\mathrm{q}-\text { good }}^{\mathrm{b}}\left(\mathcal{D}_{X}\right) .
$$


For a morphism $f: X \rightarrow Y$ of smooth algebraic varieties, we denote by $\underline{f}_{*}$ and $\underline{f}^{-1}$ the direct image and inverse image functors, respectively. Then they give

$$
\begin{aligned}
\underline{f}_{*}: & \mathrm{D}_{\mathrm{q}-\text { good }}^{\mathrm{b}}\left(\mathcal{D}_{X}\right) \rightarrow \mathrm{D}_{\mathrm{q} \text {-good }}^{\mathrm{b}}\left(\mathcal{D}_{Y}\right), \\
\underline{f}^{-1}: & \mathrm{D}_{\mathrm{q} \text {-good }}^{\mathrm{b}}\left(\mathcal{D}_{Y}\right) \rightarrow \mathrm{D}_{\mathrm{q} \text {-good }}^{\mathrm{b}}\left(\mathcal{D}_{X}\right) .
\end{aligned}
$$

Let $X_{\text {an }}$ denote the complex analytic variety associated with $X$. There is a canonical morphism $X_{\text {an }} \rightarrow X$ of $\mathbb{C}$-ringed spaces. This defines a canonical functor $(\cdot)_{\text {an }}$ : $\mathrm{D}_{\text {q-good }}^{\mathrm{b}}\left(\mathcal{D}_{X}\right) \rightarrow \mathrm{D}_{\mathrm{q} \text {-good }}^{\mathrm{b}}\left(\mathcal{D}_{X_{\text {an }}}\right)$. For a morphism $f: X \rightarrow Y$ of complex algebraic varieties we denote by $f_{\text {an }}: X_{\text {an }} \rightarrow Y_{\text {an }}$ the corresponding morphism of complex analytic varieties.

Let us take an embedding $j: X \rightarrow X^{\prime}$ from $X$ into a proper smooth algebraic variety. We call a $\mathcal{D}_{X}$-module $\mathcal{M}$ regular holonomic if $\underline{j}_{*} \mathcal{M}$ is a regular holonomic $\mathcal{D}_{X^{\prime}}$-module. We call a sheaf $F$ of $\mathbb{C}$-vector spaces on $X_{\text {an }}$ a completely $\mathbb{R}$-constructible sheaf if $\left(j_{\text {an }}\right)_{!} F$ is an $\mathbb{R}$-constructible sheaf on $X_{\text {an }}^{\prime}$. Those definitions do not depend on the embedding $j$. Let $\mathbb{R}$-cons $\left(\mathbb{C}_{X}\right)$ be the abelian category of completely $\mathbb{R}$-constructible sheaves. We denote by $\mathrm{D}_{\mathbb{R}-\mathrm{c}}^{\mathrm{b}}\left(\mathbb{C}_{X}\right)$ the full subcategory of $\mathrm{D}^{\mathrm{b}}\left(\mathbb{C}_{X_{\mathrm{an}}}\right)$ consisting of objects with completely $\mathbb{R}$-constructible cohomologies. Then $\mathrm{D}_{\mathbb{R}-\mathrm{c}}^{\mathrm{b}}\left(\mathbb{C}_{X}\right)$ is equivalent to $\mathrm{D}^{\mathrm{b}}\left(\mathbb{R}\right.$-cons $\left.\left(\mathbb{C}_{X}\right)\right)$.

We define for $F \in D_{\mathbb{R}-c}^{\mathrm{b}}\left(\mathbb{C}_{X}\right)$

$$
\begin{aligned}
\mathrm{W} \Gamma_{c}\left(X ; F \stackrel{w}{\otimes} \mathcal{O}_{X}\right) & =\Gamma\left(X_{\mathrm{an}}^{\prime} ;\left(j_{\mathrm{an}}\right) ! F \stackrel{w}{\otimes} \mathcal{O}_{X_{\mathrm{an}}^{\prime}}\right), \\
\mathrm{T} \Gamma\left(X ; \operatorname{Thom}\left(F, \mathcal{O}_{X}\right)\right) & =\Gamma\left(X_{\mathrm{an}}^{\prime} ; \operatorname{Thom}\left(\left(j_{\mathrm{an}}\right)_{!} F, \mathcal{O}_{X_{\mathrm{an}}^{\prime}}\right)\right) .
\end{aligned}
$$

More generally for $\mathcal{M} \in \mathrm{D}_{\mathrm{q}-\text { good }}^{\mathrm{b}}\left(\mathcal{D}_{X}\right)$ we set

$$
\begin{aligned}
& \mathrm{W} \Gamma_{c}\left(X ; \mathcal{H}_{\mathcal{D}}\left(\mathcal{M}, F \stackrel{w}{\otimes} \mathcal{O}_{X}\right)\right)
\end{aligned}
$$

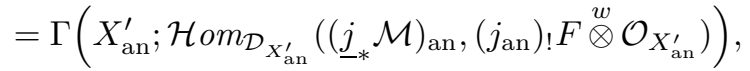

$$
\begin{aligned}
& \mathrm{T} \Gamma\left(X ; \operatorname{Thom}\left(F, \Omega_{X}\right) \otimes_{\mathcal{D}} \mathcal{M}\right) \\
& =\Gamma\left(X_{\text {an }}^{\prime} ; \operatorname{Thom}\left(\left(j_{\mathrm{an}}\right)_{!} F, \Omega_{X_{\mathrm{an}}^{\prime}}\right) \otimes_{\mathcal{D}_{X_{\mathrm{an}}^{\prime}}}\left(\underline{j}_{*} \mathcal{M}\right)_{\mathrm{an}}\right) .
\end{aligned}
$$

These definitions again do not depend on the choice of a compactification $j$.

4.2. Adjunction formulas. The adjunction formulas (3.1.9) and (3.1.10), as well as Theorem 3.3.2, hold with (not necessarily proper) smooth algebraic varieties $X$, $Y, Z$.

Consider a correspondence of smooth algebraic varieties:

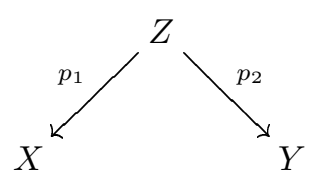

Let $F \in \mathrm{D}_{\mathbb{R}-\mathrm{c}}^{\mathrm{b}}\left(\mathbb{C}_{X}\right), \mathcal{N} \in \mathrm{D}_{\mathrm{q} \text {-good }}^{\mathrm{b}}\left(\mathcal{D}_{Y}\right), \mathcal{K}$ be a regular holonomic $\mathcal{D}_{Z}$-module, and $K=\operatorname{Sol}\left(\mathcal{K}_{\text {an }}\right)$. Then $K$ is an object of $\mathrm{D}_{\mathbb{R}-c}^{\mathrm{b}}\left(\mathbb{C}_{Z}\right)$. We set

$$
\begin{aligned}
F \circ K & =\left(p_{2 \text { an }}\right) !\left(\left(p_{1 \text { an }}\right)^{-1} F \otimes K\right), \\
\mathcal{K} \circ \mathcal{N} & =\underline{p}_{1}{ }^{*}\left(\mathcal{K} \otimes \mathcal{O} \underline{p}_{2}^{-1} \mathcal{N}\right) .
\end{aligned}
$$


Proposition 4.2.1. We have

$$
\begin{aligned}
\mathrm{W} \Gamma_{c}\left(X ; \mathcal{H o m}_{\mathcal{D}}\left(\mathcal{K} \circ \mathcal{N}, F \stackrel{w}{\otimes} \mathcal{O}_{X}\right)\right)\left[d_{X}\right] \\
\quad \simeq \mathrm{W} \Gamma_{c}\left(Y ; \mathcal{H} o m_{\mathcal{D}}\left(\mathcal{N},(F \circ K) \stackrel{w}{\otimes} \mathcal{O}_{Y}\right)\right)\left[d_{Z}\right], \\
\mathrm{T} \Gamma\left(Y ; \operatorname{Thom}\left(F \circ K, \Omega_{Y}\right) \otimes_{\mathcal{D}} \mathcal{N}\right)\left[d_{Y}\right] \\
\simeq \operatorname{T} \Gamma\left(X ; \mathcal{T h o m}\left(F, \Omega_{X}\right) \otimes_{\mathcal{D}}\left(\mathcal{K} \otimes_{\mathcal{O}} \mathcal{N}\right)\right)\left[d_{Z}\right] .
\end{aligned}
$$

Proof. We embed the correspondence (4.2.1) in another correspondence of proper algebraic varieties:

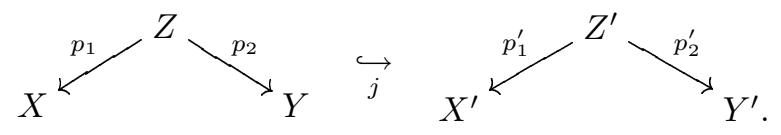

Then we have

$$
\begin{aligned}
\left(j_{\text {an } !} F\right) \circ\left(j_{\text {an }} ! K\right) & \simeq j_{\text {an } !}(F \circ K) \\
\left(\underline{j}_{!} \mathcal{K}\right) \circ\left(\underline{j}_{!} \mathcal{N}\right) & \simeq \underline{j}_{!}(\mathcal{K} \circ \mathcal{N}) .
\end{aligned}
$$

Hence we may assume from the beginning that $X, Y$ and $Z$ are proper. In such a case we can reduce the assertion to the corresponding one in the analytic setting, which is proved in [K-S2].

The same statement as in Theorem 3.3.2 holds for (not necessarily proper) smooth algebraic varieties $X, Y, Z$ in the algebraic setting, by replacing $\Gamma$ with $\mathrm{W} \Gamma_{c}$ or $\mathrm{T} \Gamma$.

\section{The LAPLACE TRANSFORM}

In this section, if there is no risk of confusion, we shall not write the symbols $\mathrm{R}$ and $\mathrm{L}$ of right and left derived functors, for short.

5.1. Fourier transform. We shall apply the results of $\S 3$ and $\S 4$ to the study of the Laplace transform. Let $V$ be an $n$-dimensional complex vector space and $V^{*}$ its dual. We regard them as complex algebraic varieties. Since a conic subanalytic set is subanalytic in a compactification of $V$, a conic $\mathbb{R}$-constructible sheaf on $V_{\text {an }}$ is completely $\mathbb{R}$-constructible. Hence we write $\mathrm{D}_{\mathbb{R}^{+}, \mathbb{R}-\mathrm{c}}^{\mathrm{b}}\left(\mathbb{C}_{V}\right)$ instead of $\mathrm{D}_{\mathbb{R}^{+}, \mathbb{R}-\mathrm{c}}^{\mathrm{b}}\left(\mathbb{C}_{V_{\text {an }}}\right)$ for short. Note that $\mathrm{D}_{\mathbb{R}^{+}, \mathbb{R}-\mathrm{c}}^{\mathrm{b}}\left(\mathbb{C}_{V}\right)$ is a full subcategory of $\mathrm{D}_{\mathbb{R}-\mathrm{c}}^{\mathrm{b}}\left(\mathbb{C}_{V}\right)$.

When there is no risk of confusion, we write $V$ instead of $V_{\text {an }}$.

We denote by $\varphi$ the function: $\varphi(z, w)=-\langle z, w\rangle$ on $V \times V^{*}$. We set, as in $\S 3$ :

$$
\begin{aligned}
A & =\left\{(z, w) \in V \times V^{*} ; \operatorname{Re} \varphi(z, w) \geq 0\right\}, \\
A^{\prime} & =\left\{(z, w) \in V \times V^{*} ; \operatorname{Re} \varphi(z, w) \leq 0\right\} .
\end{aligned}
$$

Consider the diagram:

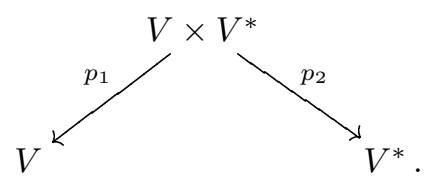


Let $F \in \mathrm{D}_{\mathbb{R}^{+}, \mathbb{R}-\mathrm{c}}^{\mathrm{b}}\left(\mathbb{C}_{V}\right)$. Its Fourier-Sato transform and inverse Fourier-Sato transform are defined by:

$$
\begin{aligned}
& F^{\wedge}=p_{2 !}\left(p_{1}^{-1} F\right)_{A}=F \circ \mathbb{C}_{A}, \\
& F^{\vee}=p_{2 !}\left(p_{1}^{!} F\right)_{A^{\prime}}=F \circ \mathbb{C}_{A^{\prime}}[2 n] .
\end{aligned}
$$

Hence $F^{\vee} \simeq F^{\wedge a}[2 n]$, where " $a$ " is the antipodal map on $V^{*}$ and $F^{\wedge a}=a_{*} F^{\wedge}$.

If $G \in \mathrm{D}_{\mathbb{R}^{+}, \mathbb{R}-\mathrm{c}}^{\mathrm{b}}\left(\mathbb{C}_{V^{*}}\right)$, we keep the same notations. For example $G^{\wedge}=p_{1 !}\left(p_{2}^{-1} G\right)_{A}$. One of the main results of the theory of the Fourier-Sato transform is that the two functors:

$$
\mathrm{D}_{\mathbb{R}^{+}, \mathbb{R}-\mathrm{c}}^{\mathrm{b}}\left(\mathbb{C}_{V}\right) \stackrel{\wedge}{\stackrel{\wedge}{\rightleftarrows}} \mathrm{D}_{\mathbb{R}^{+}, \mathbb{R}-\mathrm{c}}^{\mathrm{b}}\left(\mathbb{C}_{V^{*}}\right)
$$

are equivalences of categories inverse to each other. In particular

$$
F \simeq F^{\wedge \wedge a}[2 n] .
$$

Let $j: V \hookrightarrow P$ denote the projective compactification of $V$. For $F \in \mathrm{D}_{\mathbb{R}-\mathrm{c}}^{\mathrm{b}}\left(\mathbb{C}_{V}\right)$, we set

(5.1.2) $F \stackrel{W}{\otimes} \mathcal{O}_{V}=\mathrm{W} \Gamma_{c}\left(V ; F \stackrel{w}{\otimes} \mathcal{O}_{V}\right)=\Gamma\left(P_{\mathrm{an}} ;\left(j_{\mathrm{an}}\right) !(F) \stackrel{w}{\otimes} \mathcal{O}_{P_{\mathrm{an}}}\right)$,

(5.1.3) $\operatorname{THom}\left(F, \mathcal{O}_{V}\right)=\mathrm{T} \Gamma\left(V ; \operatorname{Thom}\left(F, \mathcal{O}_{V}\right)\right)=\Gamma\left(P_{\text {an }} ; \operatorname{Thom}\left(\left(j_{\text {an }}\right) !(F), \mathcal{O}_{P_{\text {an }}}\right)\right)$.

Let $D(V)$ denote the Weyl algebra on $V$, that is, $D(V)=\Gamma\left(V ; \mathcal{D}_{V}\right)$. The functors of formal and moderate cohomology are defined with values in $\mathrm{D}^{\mathrm{b}}(D(V))$ :

$$
\begin{array}{rll}
\cdot \stackrel{W}{\otimes} \mathcal{O}_{V} & : \quad \mathrm{D}_{\mathbb{R}-\mathrm{c}}^{\mathrm{b}}\left(\mathbb{C}_{V}\right) \rightarrow \mathrm{D}^{\mathrm{b}}(D(V)), \\
\operatorname{THom}\left(\cdot, \mathcal{O}_{V}\right) & : \quad \mathrm{D}_{\mathbb{R}-\mathrm{c}}^{\mathrm{b}}\left(\mathbb{C}_{V}\right)^{\mathrm{opp}} \rightarrow \mathrm{D}^{\mathrm{b}}(D(V)) .
\end{array}
$$

We say that a $D(V)$-module is quasi-good if it is generated by countably many elements. We denote by $\mathrm{D}_{\mathrm{q}-\text { good }}^{\mathrm{b}}(D(V))$ the full subcategory of $\mathrm{D}^{\mathrm{b}}(D(V))$ consisting of objects with quasi-good cohomology groups.

Proposition 5.1.1. The two functors

$$
\begin{aligned}
& \mathrm{D}_{\mathrm{q}-\text { good }}^{\mathrm{b}}(D(V)) \stackrel{\lambda}{\stackrel{\lambda}{\rightleftarrows}} \mathrm{D}_{\mathrm{q} \text {-good }}^{\mathrm{b}}\left(\mathcal{D}_{V}\right), \\
& \lambda(M)=\mathcal{D}_{V} \otimes_{D(V)}^{\mathrm{L}} M, \\
& \gamma(\mathcal{M})=\mathrm{R} \Gamma(V ; \mathcal{M}),
\end{aligned}
$$

are well-defined and inverse to each other.

Proof. Since $V$ is affine, the category of quasi-good $D(V)$-modules is equivalent to that of quasi-good $\mathcal{D}_{V}$-modules. Moreover $\mathrm{D}_{\mathrm{q} \text {-good }}^{\mathrm{b}}(D(V))$ and $\mathrm{D}_{\mathrm{q} \text {-good }}^{\mathrm{b}}\left(\mathcal{D}_{V}\right)$ are their derived categories.

We denote by $\wedge$ the Fourier isomorphism:

$$
\wedge: D(V) \stackrel{\sim}{\rightarrow} D\left(V^{*}\right) .
$$

If $\left(z_{1}, \ldots, z_{n}\right)$ is a system of linear coordinates on $V$ and $\left(w_{1}, \ldots, w_{n}\right)$ the dual coordinate system on $V^{*}$, then $\wedge$ is given by:

$$
\begin{aligned}
\left(z_{j}\right)^{\wedge} & =-\frac{\partial}{\partial w_{j}} \\
\left(\frac{\partial}{\partial z_{j}}\right)^{\wedge} & =w_{j} .
\end{aligned}
$$


Let $\vee: D\left(V^{*}\right) \rightarrow D(V)$ be the inverse of $\wedge$. For a $D\left(V^{*}\right)$-module $N$, the ring isomorphism $\wedge: D(V) \stackrel{\sim}{\rightarrow} D\left(V^{*}\right)$ makes $N$ a $D(V)$-module, which we denote by $N^{\wedge}$. Thus it gives an equivalence of categories $\wedge: \mathrm{D}_{\mathrm{q} \text {-good }}^{\mathrm{b}}\left(D\left(V^{*}\right)\right) \rightarrow \mathrm{D}_{\mathrm{q} \text {-good }}^{\mathrm{b}}(D(V))$, and similarly $\vee: \mathrm{D}_{\mathrm{q} \text {-good }}^{\mathrm{b}}(D(V)) \rightarrow \mathrm{D}_{\mathrm{q} \text {-good }}^{\mathrm{b}}\left(D\left(V^{*}\right)\right)$.

Let us come back to the function $\varphi(z, w)=-\langle z, w\rangle$ on $V \times V^{*}$, and set as in $\S 3$ :

$$
\begin{aligned}
\mathcal{L} & =\mathcal{D}_{V \times V^{*}} e^{\varphi}, \\
\mathcal{L}^{\prime} & =\mathcal{D}_{V \times V^{*}} e^{-\varphi} .
\end{aligned}
$$

We have the following result due to Katz-Laumon [K-L] (see also $[\mathrm{M}]$ ).

Proposition 5.1.2. There are isomorphisms functorial in $M \in \mathrm{D}_{\mathrm{q}-\text { good }}^{\mathrm{b}}(D(V))$ and $N \in \mathrm{D}_{\mathrm{q}-\text { good }}^{\mathrm{b}}\left(D\left(V^{*}\right)\right)$ :

$$
\begin{aligned}
\lambda(M) \circ \mathcal{L}^{\prime} & \simeq \lambda\left(M^{\vee}\right), \\
\mathcal{L} \circ \lambda(N) & \simeq \lambda\left(N^{\wedge}\right) .
\end{aligned}
$$

5.2. The Laplace transform. We are now ready to apply the results of $\S \S 3-4$.

Let $F \in \mathrm{D}_{\mathbb{R}^{+}, \mathbb{R}-\mathrm{c}}^{\mathrm{b}}\left(\mathbb{C}_{V}\right)$ and let $N \in \mathrm{D}_{\text {q-good }}^{\mathrm{b}}\left(D\left(V^{*}\right)\right)$. We shall use the kernels $\mathcal{L}$ and $\mathcal{L}^{\prime}$ defined in (5.1.6) and (5.1.7).

By Propositions 5.1.1 and 5.1.2, $\lambda(N) \in \mathrm{D}_{\text {q-good }}^{\mathrm{b}}\left(\mathcal{D}_{V^{*}}\right)$ and $\mathcal{L} \circ \lambda(N) \simeq \lambda\left(N^{\wedge}\right)$. Hence (3.1.11) defines the morphism:

$$
\operatorname{Hom}_{D(V)}\left(N^{\wedge}, F \stackrel{W}{\otimes} \mathcal{O}_{V}\right) \underset{L}{\longrightarrow} \operatorname{Hom}_{D\left(V^{*}\right)}\left(N, F^{\wedge}[n] \stackrel{W}{\otimes} \mathcal{O}_{V^{*}}\right)
$$

Similarly, (3.1.12) defines:

$$
\operatorname{THom}\left(F^{\wedge}[n], \Omega_{V^{*}}\right) \otimes_{D\left(V^{*}\right)} N \underset{{ }^{t} L}{\longrightarrow} \operatorname{THom}\left(F, \Omega_{V}\right) \otimes_{D(V)} N^{\wedge}
$$

We call $L$ and ${ }^{t} L$ the Laplace morphisms.

Using $\mathcal{L}^{\prime}$, one constructs similarly for $G \in \mathrm{D}_{\mathbb{R}^{+}, \mathbb{R}-\mathrm{c}}^{\mathrm{b}}\left(\mathbb{C}_{V^{*}}\right)$ and $M \in \mathrm{D}_{\mathrm{q} \text {-good }}^{\mathrm{b}}(D(V))$ the morphisms

$$
\begin{array}{rll}
\operatorname{Hom}_{D\left(V^{*}\right)}\left(M^{\vee}, G \stackrel{W}{\otimes} \mathcal{O}_{V^{*}}\right) & \underset{L^{\prime}}{\longrightarrow} & \operatorname{Hom}_{D(V)}\left(M, G^{\wedge a}[n] \stackrel{W}{\otimes} \mathcal{O}_{V}\right), \\
\operatorname{THom}\left(G^{\wedge a}[n], \Omega_{V}\right) \otimes_{D(V)} M & \underset{{ }^{t} L^{\prime}}{\longrightarrow} & \operatorname{THom}\left(G, \Omega_{V^{*}}\right) \otimes_{D\left(V^{*}\right)} M^{\vee} .
\end{array}
$$

We call $L^{\prime}$ and ${ }^{t} L^{\prime}$ the inverse Laplace morphisms.

By combining the above morphisms, we obtain

$$
\begin{array}{rll}
\operatorname{Hom}_{D(V)}\left(M^{\vee \wedge}, F \otimes \mathcal{O}_{V}\right) & \longrightarrow & \operatorname{Hom}_{D\left(V^{*}\right)}\left(M^{\vee}, F^{\wedge}[n] \otimes \mathcal{O}_{V^{*}}\right) \\
& \underset{L^{\prime}}{\longrightarrow} & \operatorname{Hom}_{D(V)}\left(M, F^{\wedge \wedge a}[2 n] \otimes \mathcal{O}_{V}\right), \\
\operatorname{THom}\left(F^{\wedge \wedge a}[n], \Omega_{V}\right) \otimes_{D(V)} M & \underset{{ }^{{ }_{L^{\prime}}}}{\longrightarrow} & \operatorname{THom}\left(F^{\wedge}[n], \Omega_{V^{*}}\right) \otimes_{D\left(V^{*}\right)} M^{\vee} \\
& \underset{{ }^{t} L}{ } & \operatorname{THom}\left(F, \Omega_{V}\right) \otimes_{D(V)} M^{\vee \wedge} .
\end{array}
$$

Theorem 5.2.1. Let us identify $F^{\wedge \wedge a}[2 n]$ with $F$ and $M$ with $M^{\vee \wedge}$. Then $L$ and $L^{\prime}$, as well as ${ }^{t} L$ and ${ }^{t} L^{\prime}$, are inverse to each other, i.e.

$$
\begin{aligned}
& L^{\prime} \circ L=\mathrm{id}, \quad{ }^{t} L \circ{ }^{t} L^{\prime}=\mathrm{id}, \\
& L \circ L^{\prime}=\text { id, } \quad{ }^{t} L^{\prime} \circ{ }^{t} L=\text { id . }
\end{aligned}
$$


Proof. We shall apply Theorem 3.3.2 in its algebraic setting.

With the notations of this theorem, we have

$$
\begin{aligned}
& X=V, Y=V^{*}, Z=V, \\
& S_{1}=S_{2}=S_{3}=\emptyset \\
& \varphi_{1}(z, w)=-\langle z, w\rangle, \varphi_{2}\left(w, z^{\prime}\right)=\left\langle z^{\prime}, w\right\rangle, \varphi_{0}\left(z, w, z^{\prime}\right)=\left\langle-z+z^{\prime}, w\right\rangle, \\
& A_{1}=A, A_{2}=A^{\prime}, A_{0}=\left\{\left(z, w, z^{\prime}\right) \in V \times V^{*} \times V ; \operatorname{Re}\left\langle z-z^{\prime}, w\right\rangle \leq 0\right\}, \\
& A_{3}=\left\{\left(z, w, z^{\prime}\right) \in V \times V^{*} \times V ; \operatorname{Re}\langle z, w\rangle \leq 0, \operatorname{Re}\left\langle z^{\prime}, w\right\rangle \geq 0\right\} \\
& \mathcal{L}_{1}=\mathcal{L}, \mathcal{L}_{2}=\mathcal{L}^{\prime}, \mathcal{L}_{0}=\mathcal{D}_{V \times V^{*} \times V} e^{\varphi_{0}}, \mathcal{L}_{0}^{\prime}=\mathcal{D}_{V \times V^{*} \times V} e^{-\varphi_{0}} .
\end{aligned}
$$

We denote by $r_{1}, r_{2}, r_{3}$ the projections from $V \times V^{*} \times V$ to $V \times V^{*}, V^{*} \times V, V \times V$ as in the diagram (3.3.1).

Let $\Delta_{V}$ denote the diagonal of $V \times V$, and let $\mathcal{K}$ be the $\mathcal{D}_{V \times V^{*}-\text { module }} \mathcal{B}_{\Delta_{V} \mid V \times V}$. We have

$$
K=\operatorname{Sol}\left(\mathcal{K}_{\text {an }}\right) \simeq \mathbb{C}_{\Delta_{V}}[-n] .
$$

We denote by $k$ the embedding $r_{3}^{-1} \Delta_{V} \hookrightarrow V \times V^{*} \times V$. We have:

$$
\begin{aligned}
& \underline{k}^{-1} \mathcal{L}_{0} \simeq \mathcal{O}_{r_{3}^{-1} \Delta_{V}}, \\
& \underline{k}_{*} \mathcal{O}_{r_{3}^{-1} \Delta_{V}} \simeq \underline{r}_{3}^{-1} \mathcal{K} .
\end{aligned}
$$

Hence the natural morphism

$$
\underline{k}_{*} \underline{k}^{-1} \mathcal{L}_{0}[-n] \rightarrow \mathcal{L}_{0}
$$

defines:

$$
\underline{r}_{3}^{-1} \mathcal{K}[-n] \rightarrow \mathcal{L}_{0}
$$

On the other hand, we have:

$$
\begin{aligned}
\underline{r}_{3}^{-1} \mathcal{K} \otimes_{\mathcal{O}} \mathcal{L}_{0}^{\prime} & \simeq\left(\underline{k}_{*} \mathcal{O}_{r_{3}^{-1} \Delta_{V}}\right) \otimes_{\mathcal{O}} \mathcal{L}_{0}^{\prime} \\
& \simeq \underline{k}_{*}\left(\mathcal{O}_{r_{3}^{-1} \Delta_{V}} \otimes_{\mathcal{O}} \underline{k}^{-1} \mathcal{L}_{0}^{\prime}\right) \\
& \simeq \underline{k}_{*} \mathcal{O}_{r_{3}^{-1} \Delta_{V}} .
\end{aligned}
$$

Hence, the hypothesis (3.3.10) of Theorem 3.3.2 is satisfied. For $z, z^{\prime} \in V, r_{3}^{-1}\left(z, z^{\prime}\right)$ $\cap A_{0}$ is a closed half-space if $z \neq z^{\prime}$, and is isomorphic to $\mathbb{R}^{2 n}$ if $z=z^{\prime}$. We get

$$
r_{3 !} \mathbb{C}_{A_{0}} \simeq \mathbb{C}_{\Delta_{V}}[-2 n] \simeq K[-n],
$$

and the morphism $\mathbb{C}_{A_{0}} \rightarrow \mathbb{C}_{A_{3}}$ induces

$$
K[-n] \rightarrow r_{3 !} \mathbb{C}_{A_{3}}
$$

Hence all the hypotheses of Theorem 3.3.2 are satisfied.

To conclude, we remark that:

- if $\mathcal{N} \in \mathrm{D}_{\text {q-good }}^{\mathrm{b}}\left(\mathcal{D}_{V}\right)$, then $\mathcal{K} \circ \mathcal{N} \simeq \mathcal{N}$.

- for $F \in \mathrm{D}_{\mathbb{R}^{+}, \mathbb{R}-\mathrm{c}}^{\mathrm{b}}\left(\mathbb{C}_{V}\right)$, the morphism $F \simeq F \circ K[n] \simeq F \circ r_{3 !} \mathbb{C}_{A_{0}}[2 n] \rightarrow$ $F \circ r_{3 !} \mathbb{C}_{A_{3}}[2 n] \simeq F \circ \mathbb{C}_{A_{1}} \circ \mathbb{C}_{A_{2}}[2 n] \simeq F^{\wedge \wedge a}[2 n]$ coincides with the isomorphism $F \stackrel{\sim}{\rightarrow} F^{\wedge \wedge a}[2 n]$ constructed in $[\mathrm{K}-\mathrm{S} 1, \mathrm{Ch} . \mathrm{III}]$.

This completes the proof. 
Remark 5.2.2. The Laplace transform commutes with duality. Denote by $D$ the duality functor of topological vector spaces of type $F N$ and $D F N$ :

$$
\mathrm{D}^{\mathrm{b}}(F N)^{\mathrm{opp}} \underset{D}{\stackrel{D}{\rightleftarrows}} \mathrm{D}^{\mathrm{b}}(D F N)
$$

Then the functor $D$ sends

$$
\operatorname{Hom}_{D(V)}\left(N^{\wedge}, F \stackrel{W}{\otimes} \mathcal{O}_{V}\right) \underset{L}{\stackrel{\sim}{\rightarrow}} \operatorname{Hom}_{D\left(V^{*}\right)}\left(N, F^{\wedge}[n] \stackrel{W}{\otimes} \mathcal{O}_{V^{*}}\right)
$$

to

$$
\operatorname{THom}\left(F, \Omega_{V}[n]\right) \otimes_{D(V)} N^{\wedge} \underset{{ }_{L}}{\tilde{\tau}} \operatorname{THom}\left(F^{\wedge}[n], \Omega_{V^{*}}[n]\right) \otimes_{D\left(V^{*}\right)} N
$$

The proof follows easily from the constructions.

We shall compare our construction with the classical Fourier transform. Let $V_{\mathbb{R}}$ be a real vector space and set $V=\mathbb{C} \otimes_{\mathbb{R}} V_{\mathbb{R}}$. If we take $\mathbb{C}_{V_{\mathbb{R}}}[-n]$ as $F$ in (5.2.2), we have $F^{\wedge}[n] \simeq \mathbb{C}_{\sqrt{-1} V_{\mathbb{R}}^{*}}[-n]$ and we obtain the isomorphism

$$
\begin{array}{ccc}
{ }^{t} L: \operatorname{THom}\left(\mathbb{C}_{\sqrt{-1} V_{\mathbb{R}}^{*}}[-n], \mathcal{O}_{V^{*}}\right) & \stackrel{\sim}{\longrightarrow} \operatorname{THom}\left(\mathbb{C}_{V_{\mathbb{R}}}[-n], \mathcal{O}_{V}\right) \\
\mathcal{S}^{\prime}\left(\sqrt{-1} V_{\mathbb{R}}^{*}\right) & \mathcal{S}^{\prime}\left(V_{\mathbb{R}}\right) .
\end{array}
$$

Proposition 5.2.3. The above isomorphism coincides with the classical Fourier transform of Schwartz's tempered distributions

$$
u(w) \mapsto \hat{u}(z)=\int_{\sqrt{-1} V_{\mathbb{R}}^{*}} u(w) e^{\langle z, w\rangle} d w \in \mathcal{S}^{\prime}\left(V_{\mathbb{R}}\right) .
$$

The proof will be given in the appendix.

5.3. Generalization to vector bundles. The Laplace transform on a vector space constructed above can be generalized to vector bundles.

Let $X$ be a complex analytic variety. Let $\tau: V \rightarrow X$ be a vector bundle with fiber dimension $n$, and $\pi: V^{*} \rightarrow X$ its dual vector bundle. Let us denote by $D_{V}$ the sheaf of rings on $X$ of differential operators on $V$ with polynomial coefficients on the fibers.

In order to describe the Fourier transform of $D_{V}$-modules, we consider the line bundle on $X$ :

$$
\operatorname{det}(V)=\bigwedge^{n} \mathcal{O}_{X}(V)
$$

where $\mathcal{O}_{X}(V)$ denotes the sheaf on $X$ of sections of $V$.

We have:

$$
\Omega_{V^{*} / X} \simeq \pi^{*} \operatorname{det}(V) .
$$

Let $(x, z)$ denote a local coordinate system on $V$, linear in the fibers, and let $(x, w)$ denote the dual coordinates on $V^{*}$. The kernel:

$$
K\left(x, x^{\prime}, z, w\right)=\exp (-\langle z, w\rangle) \delta\left(x-x^{\prime}\right) d x^{\prime}
$$

is a section of the sheaf $H_{\left[V \times V_{X}^{*}\right]}^{d_{X}}\left(\mathcal{O}_{V \times V^{*}}\right) \otimes_{p_{2}^{-1} \mathcal{O}_{X}} p_{2}^{-1} \Omega_{X}$, where $p_{2}$ is the second projection $V \times V^{*} \rightarrow X$. This kernel does not depend on the local coordinate system and is globally defined on $V \times V^{*}$.

The correspondence $P \mapsto \stackrel{X}{Q}$ given by the relation

$$
P\left(x, z, \partial_{x}, \partial_{z}\right) K=Q\left(x, w, \partial_{x}, \partial_{w}\right) K
$$


defines a homomorphism

$$
\begin{aligned}
\Omega_{X} \otimes_{\mathcal{O}} \tau_{*} \mathcal{D}_{V} \otimes_{\mathcal{O}} \Omega_{X}^{\otimes-1} & \rightarrow \pi_{*}\left(\Omega_{V^{*}} \otimes_{\mathcal{O}} \mathcal{D}_{V^{*}} \otimes_{\mathcal{O}} \Omega_{V^{*}}^{\otimes-1}\right)^{\mathrm{opp}} \\
& \rightarrow \pi_{*}\left(\Omega_{V^{*} / X} \otimes_{\mathcal{O}} \mathcal{D}_{V^{*}} \otimes_{\mathcal{O}} \Omega_{V^{*} / X}^{\otimes-1}\right)
\end{aligned}
$$

This induces an isomorphism:

$$
D_{V} \simeq \operatorname{det}(V) \otimes_{\mathcal{O}} D_{V^{*}} \otimes_{\mathcal{O}} \operatorname{det}(V)^{\otimes-1} .
$$

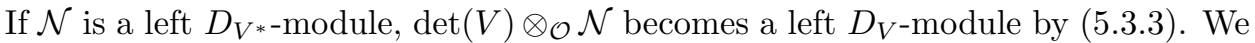
denote it by $\mathcal{N}^{\wedge}$ :

$$
\mathcal{N}^{\wedge}=\operatorname{det}(V) \otimes_{\mathcal{O}} \mathcal{N} \quad \text { as a } D_{V^{-}} \text {module. }
$$

In order to state the Laplace inversion formula on vector bundles, we introduce some notations. Let $\bar{\tau}: P \rightarrow X$ and $\bar{\pi}: P^{*} \rightarrow X$ denote the projective compactifications of $V$ and $V^{*}$, respectively. Let $F \in \mathrm{D}_{\mathbb{R}^{+}, \mathbb{R}-\mathrm{c}}^{\mathrm{b}}\left(\mathbb{C}_{V}\right)$. We set:

$$
\begin{aligned}
F \stackrel{W}{\otimes} \mathcal{O}_{V} & =\bar{\tau}_{*}\left(j_{!} F \stackrel{w}{\otimes} \mathcal{O}_{P}\right), \\
\operatorname{THom}\left(F, \mathcal{O}_{V}\right) & =\bar{\tau}_{*}\left(\operatorname{Thom}\left(j ! F, \mathcal{O}_{P}\right)\right),
\end{aligned}
$$

where $j: V \hookrightarrow P$ is the embedding. These definitions are a generalization of (5.1.2) and (5.1.3) to the vector bundle case.

We define the Laplace transforms $L$ and ${ }^{t} L$ by formulas (3.1.11) and (3.1.12) with $\varphi=\exp (-\langle z, w\rangle)$, and similarly for $L^{\prime}$ and ${ }^{t} L^{\prime}$, associated with $-\varphi$.

Theorem 5.3.1. Let $\mathcal{N} \in \mathrm{D}_{\mathrm{q}-\operatorname{good}}^{\mathrm{b}}\left(D_{V^{*}}\right)$ and let $F \in \mathrm{D}_{\mathbb{R}^{+}, \mathbb{R}-\mathrm{c}}^{\mathrm{b}}\left(\mathbb{C}_{V}\right)$. Then the Laplace transforms induce isomorphisms in $\mathrm{D}^{\mathrm{b}}\left(\mathbb{C}_{X}\right)$ :

$$
\begin{array}{rcl}
\mathcal{H o m}_{D_{V}}\left(\mathcal{N}^{\wedge}, F \stackrel{W}{\otimes} \mathcal{O}_{V}\right) & \underset{L}{\stackrel{\sim}{c}} & \mathcal{H o m}_{D_{V^{*}}}\left(\mathcal{N}, F^{\wedge}[n] \stackrel{W}{\otimes} \mathcal{O}_{V^{*}}\right), \\
\operatorname{THom}\left(F, \Omega_{V}\right) \otimes_{D_{V}} \mathcal{N}^{\wedge} & \underset{{ }^{t} L}{\check{L}} & \operatorname{THom}\left(F^{\wedge}[n], \Omega_{V^{*}}\right) \otimes_{D_{V^{*}}} \mathcal{N} .
\end{array}
$$

Moreover $L^{\prime}\left(\right.$ resp. $\left.{ }^{t} L^{\prime}\right)$ is the inverse to $L\left(\right.$ resp. $\left.{ }^{t} L\right)$.

\section{Applications}

As above we denote by $V$ an $n$-dimensional complex vector space, by $V^{*}$ its dual and by $j: V \hookrightarrow P$ its projective compactification.

If $Z$ is a locally closed subset of $V$ subanalytic in $P$, we set:

$$
\begin{aligned}
\mathrm{R} \Gamma_{[Z]}\left(V ; \mathcal{O}_{V}\right) & =\operatorname{THom}\left(\mathbb{C}_{Z}, \mathcal{O}_{V}\right)=\mathrm{R} \Gamma\left(P ; \operatorname{Thom}\left(j ! \mathbb{C}_{Z}, \mathcal{O}_{P}\right)\right), \\
H_{[Z]}^{p}\left(V ; \mathcal{O}_{V}\right) & =H^{p}\left(\mathrm{R} \Gamma_{[Z]}\left(V ; \mathcal{O}_{V}\right)\right) .
\end{aligned}
$$

Hence $H_{[Z]}^{p}\left(V ; \mathcal{O}_{V}\right)$ denotes the "moderate cohomology" of $\mathcal{O}$ supported by $Z$. In particular, if $U$ is an open subanalytic subset, $H_{[U]}^{0}\left(V ; \mathcal{O}_{V}\right)$ is the subspace of $\Gamma\left(U ; \mathcal{O}_{V}\right)$ of holomorphic functions with tempered growth at the boundary of $U$ including infinity. 
6.1. Convex cones. Let $\gamma$ be a convex cone in $V$. We set:

$$
\begin{aligned}
\gamma^{\circ} & =\left\{w \in V^{*} ; \operatorname{Re}\langle z, w\rangle \geq 0 \quad \text { for all } z \in \gamma\right\}, \\
\gamma^{a} & =-\gamma, \\
\text { Int } \gamma & =\text { the interior of } \gamma .
\end{aligned}
$$

We keep the same notations on $V^{*}$. If $\gamma$ is an open convex cone, one has:

$$
\left(\mathbb{C}_{\gamma}\right)^{\wedge}=\mathbb{C}_{\gamma^{\circ a}}[-2 n] .
$$

If $\gamma$ is closed and proper (i.e. $\gamma$ contains no line), then:

$$
\left(\mathbb{C}_{\gamma}\right)^{\wedge} \simeq \mathbb{C}_{\text {Int } \gamma^{\circ}}
$$

Let $U$ be a convex open subanalytic cone in $V$, and $Z=U^{\circ a}$. Applying Theorem 5.2.1, we find the Laplace isomorphism:

$$
\mathrm{R} \Gamma_{[U]}\left(V ; \mathcal{O}_{V}\right) \underset{L}{\stackrel{\sim}{\longrightarrow}} \mathrm{R} \Gamma_{[Z]}\left(V^{*} ; \mathcal{O}_{V^{*}}\right)[n] .
$$

Proposition 6.1.1. For $U$ and $Z$ as above, we have

and

$$
\begin{aligned}
& H_{[U]}^{j}\left(V ; \mathcal{O}_{V}\right)=0 \quad \text { for } j \neq 0, \\
& H_{[Z]}^{j}\left(V^{*} ; \mathcal{O}_{V^{*}}\right)=0 \quad \text { for } j \neq n,
\end{aligned}
$$

$$
H_{[U]}^{0}\left(V ; \mathcal{O}_{V}\right) \underset{L}{\stackrel{\sim}{\longrightarrow}} H_{[Z]}^{n}\left(V^{*} ; \mathcal{O}_{V^{*}}\right) .
$$

Proof. The left hand side of (6.1.3) is concentrated in degree $\geq 0$ and the right hand side in degree $\leq 0$. Hence both sides are concentrated in degree 0 .

Similarly, one gets:

$$
\mathbb{C}_{U} \stackrel{W}{\otimes} \mathcal{O}_{V}[n] \underset{L}{\stackrel{\sim}{\longrightarrow}} \mathbb{C}_{Z} \stackrel{W}{\otimes} \mathcal{O}_{V^{*}}
$$

and both sides are concentrated in degree 0 .

Now let $V=\mathbb{C} \otimes_{\mathbb{R}} V_{\mathbb{R}}$ be the complexification of a real vector space $V_{\mathbb{R}}$. We have (see [K-S2, Th. 5.10])

$$
\operatorname{THom}\left(\mathbb{C}_{V_{\mathbb{R}}}[-n], \mathcal{O}_{V}\right) \simeq \mathcal{S}^{\prime}\left(V_{\mathbb{R}}\right),
$$

the space of tempered distributions on $V_{\mathbb{R}}$, and

$$
\mathbb{C}_{V_{\mathbb{R}}} \stackrel{W}{\otimes} \mathcal{O}_{V} \simeq \mathcal{S}\left(V_{\mathbb{R}}\right)
$$

the space of rapidly decreasing $C^{\infty}$-functions on $V_{\mathbb{R}}$.

Let $\gamma($ resp. $\lambda$ ) be a closed (resp. open) subanalytic convex cone contained in $V_{\mathbb{R}}$. We set

$$
\begin{aligned}
\Gamma_{\gamma}\left(\mathcal{S}^{\prime}\left(V_{\mathbb{R}}\right)\right) & =\operatorname{THom}\left(\mathbb{C}_{\gamma}[-n], \mathcal{O}_{V}\right), \\
\mathcal{S}^{\prime}(\lambda) & =\operatorname{THom}\left(\mathbb{C}_{\lambda}[-n], \mathcal{O}_{V}\right), \\
\mathcal{S}(\gamma) & =\mathbb{C}_{\gamma}{ }^{W} \mathcal{O}_{V}, \\
\Gamma_{\lambda} \mathcal{S}\left(V_{\mathbb{R}}\right) & =\mathbb{C}_{\lambda} \stackrel{W}{\otimes} \mathcal{O}_{V} .
\end{aligned}
$$

Hence $\Gamma_{\gamma}\left(\mathcal{S}^{\prime}\left(V_{\mathbb{R}}\right)\right)$ is the space of tempered distributions supported by $\gamma, \mathcal{S}^{\prime}(\lambda)$ is the space of tempered distributions on $\lambda, \mathcal{S}(\gamma)$ is the space of Whitney functions on 
$\gamma$ vanishing up to infinite order at infinity, and $\Gamma_{\lambda} \mathcal{S}\left(V_{\mathbb{R}}\right)$ is the subspace of $\mathcal{S}\left(V_{\mathbb{R}}\right)$ of functions vanishing up to infinite order on $V_{\mathbb{R}} \backslash \lambda$.

The Laplace transform induces the isomorphisms:

$$
\begin{aligned}
& \Gamma_{\gamma} \mathcal{S}^{\prime}\left(V_{\mathbb{R}}\right) \simeq H_{\left[\operatorname{Int} \gamma^{\circ}\right]}^{0}\left(V^{*} ; \mathcal{O}_{V^{*}}\right), \\
& \mathcal{S}^{\prime}(\lambda) \simeq H_{\left[\lambda^{\circ a}\right]}^{n}\left(V^{*} ; \mathcal{O}_{V^{*}}\right), \\
& \mathcal{S}(\gamma) \simeq \mathbb{C}_{\text {Int } \gamma^{\circ}}[n] \stackrel{W}{\otimes} \mathcal{O}_{V^{*}}, \\
& \Gamma_{\lambda} \mathcal{S}\left(V_{\mathbb{R}}\right) \simeq \mathbb{C}_{\lambda \circ a} \stackrel{W}{\otimes} \mathcal{O}_{V^{*}} .
\end{aligned}
$$

Among those isomorphisms, it is a well-known result that the Laplace transform interchanges tempered distributions supported by $\gamma$ and tempered holomorphic functions in the dual tube.

Remark 6.1.2. Let $F \in \mathbb{R}$-cons $\left(\mathbb{C}_{V_{\mathbb{R}}}\right)$, and denote by $i$ the embedding $V_{\mathbb{R}} \hookrightarrow V$. Since $\operatorname{THom}\left(i_{!} F[-n], \mathcal{O}_{V}\right)$ is concentrated in degree 0 (see Example 2.2.1), we get

$\operatorname{THom}\left(p^{-1} F^{\wedge}, \mathcal{O}_{V^{*}}\right)$ is concentrated in degree 0 ,

where $p: V^{*} \rightarrow V_{\mathbb{R}}^{*}$ is the transpose of $i$, and $F^{\wedge}$ is the Fourier-Sato transform on $V_{\mathbb{R}}$. Using the fact that $F \mapsto \operatorname{THom}\left(i_{!} F[-n], \mathcal{O}_{V}\right)$ is exact, one may deduce various results, such as the well-known "Edge of the wedge theorem" of Martineau [Mr] in the tempered setting. Details are left to the reader.

6.2. Quadratic cones. Let $V_{\mathbb{R}}$ be an $n$-dimensional real vector space, $V$ its complexification, $z=\left(z_{1}, \ldots, z_{n}\right)$ a system of linear coordinates on $V$, with $z=$ $x+\sqrt{-1} y$, and $w=\left(w_{1}, \ldots, w_{n}\right)$ the dual coordinates on $V^{*}$ with $w=u+\sqrt{-1} v$. Let $p, q$ be integers with $p, q \geq 1$ and $p+q=n$. We write $z=\left(z^{\prime}, z^{\prime \prime}\right)$ where

$$
z^{\prime}=\left(z_{1}, \ldots, z_{p}\right), \quad z^{\prime \prime}=\left(z_{p+1}, \ldots, z_{n}\right) .
$$

We use similar notations such as $x=\left(x^{\prime}, x^{\prime \prime}\right), w=\left(w^{\prime}, w^{\prime \prime}\right)$, etc. We consider the solid quadratic cones:

$$
\begin{aligned}
& \gamma=\left\{z \in V ; y=0, x^{\prime 2}-x^{\prime \prime 2} \geq 0\right\} \\
& \lambda=\left\{w \in V^{*} ; u^{\prime 2}-u^{\prime \prime 2} \leq 0\right\} .
\end{aligned}
$$

Lemma 6.2.1. We have:

$$
\mathbb{C}_{\gamma}^{\wedge} \simeq \mathbb{C}_{\lambda}[-p]
$$

Before proving this lemma, let us discuss its applications. Applying Theorem 5.2.1, we find the following result.

Proposition 6.2.2. The Laplace transform induces an isomorphism

$$
\Gamma_{\gamma}\left(\mathcal{S}^{\prime}\left(V_{\mathbb{R}}\right)\right) \underset{L}{\stackrel{\sim}{\longrightarrow}} H_{[\lambda]}^{p}\left(V^{*} ; \mathcal{O}_{V^{*}}\right)
$$

and $H_{[\lambda]}^{j}\left(V^{*} ; \mathcal{O}_{V^{*}}\right)=0$ for $j \neq p$.

Remark 6.2.3. The Laplace transform of tempered distributions supported by the solid quadratic cone $\gamma$ has already been considered by Faraut-Gindikin [F-G] and the formula (6.2.1) should be considered due to them, although their formulation and proof are quite different from ours. 
Proof of Lemma 6.2.1. For $u \in V_{\mathbb{R}}^{*}$, set:

$$
\gamma_{u}=\{x \in \gamma ;\langle x, u\rangle \leq 0\} .
$$

Then $\left(\mathbb{C}_{\gamma}^{\wedge}\right)_{w} \simeq \mathrm{R} \Gamma_{c}\left(V_{\mathbb{R}} ; \mathbb{C}_{\gamma_{u}}\right)$ with $u=\operatorname{Re} w$. We have (see [K-S1, Ex III 5]):

$$
\mathrm{R} \Gamma_{c}\left(V_{\mathbb{R}} ; \mathbb{C}_{\gamma}\right) \simeq \mathbb{C}[-p] .
$$

Hence, it is enough to check the following statements:

(i) if $u \notin \lambda, \mathrm{R} \Gamma_{c}\left(V_{\mathbb{R}} ; \mathbb{C}_{\gamma_{u}}\right)=0$,

(ii) if $u \in \lambda \backslash\{0\}$, the morphism

$$
\mathrm{R} \Gamma_{c}\left(V_{\mathbb{R}} ; \mathbb{C}_{\gamma}\right) \longrightarrow \mathrm{R} \Gamma_{c}\left(V_{\mathbb{R}} ; \mathbb{C}_{\gamma_{u}}\right)
$$

is an isomorphism.

Let us prove (i). Let $u=\left(u^{\prime}, u^{\prime \prime}\right)$. We may assume $u^{\prime \prime}=0$. Let $f$ be the projection $\mathbb{R}^{p} \times \mathbb{R}^{q} \rightarrow \mathbb{R}^{p},\left(x^{\prime}, x^{\prime \prime}\right) \mapsto x^{\prime}$, and set $\tilde{\gamma}_{u}=f\left(\gamma_{u}\right)$. The fibers of $f$ above $\tilde{\gamma}_{u}$ are closed balls and $\tilde{\gamma}_{u}$ is a closed half plane. Hence $\mathrm{R} \Gamma_{c}\left(\tilde{\gamma}_{u} ; \mathbb{C}_{\tilde{\gamma}_{u}}\right)=0$, and (i) follows. Let us prove (ii). We may assume $u=(0, \ldots, 0,1)$. Let $f$ be the projection $\mathbb{R}^{n} \rightarrow \mathbb{R}^{n-1}, x \mapsto\left(x_{1}, \ldots, x_{n-1}\right)$. Set $\tilde{\gamma}_{u}^{-}=f\left(\gamma \backslash \gamma_{u}\right)$. Then the fibers of $f$ above $\tilde{\gamma}_{u}^{-}$are intervals $(0, a]$ for some $a \in \mathbb{R}$. Hence $\mathrm{R} f_{!} \mathbb{C}_{\gamma \backslash \gamma_{u}}=0$, and we obtain $\mathrm{R} \Gamma_{c}\left(M ; \mathbb{C}_{\gamma \backslash \gamma_{u}}\right)=0$, which implies (ii).

6.3. The sheaf $\mathcal{O}_{V}^{t}$. Let $V$ be an $n$-dimensional complex vector space. For two open cones $U_{1}$ and $U_{2}$, we write for short

$$
U_{1} \Subset U_{2}
$$

if $\overline{U_{1}} \subset U_{2} \cup\{0\}$. We shall construct conic sheaves associated with conic presheaves. Let $\mathcal{T}$ be a family of open cones satisfying:

$$
\left\{\begin{array}{l}
\text { for each } z \in V, \text { and each open conic neighborhood } U \text { of } z, \\
\text { there exists } U^{\prime} \in \mathcal{T} \text { with } z \in U^{\prime} \subset U .
\end{array}\right.
$$

Let $\mathcal{G}$ be a presheaf of $\mathbb{C}$-vector spaces on $\mathcal{T}$. The classical construction of a sheaf associated to a presheaf gives a conic sheaf $\tilde{\mathcal{G}}$ and a morphism of presheaves $\theta: \mathcal{G} \rightarrow \tilde{\mathcal{G}}$ such that any morphism $\mathcal{G} \rightarrow \mathcal{F}$ with a conic sheaf $\mathcal{F}$ factorizes through $\theta$.

Let $j: V \hookrightarrow P$ denote, as above, the projective compactification of $V$. For $F \in \mathrm{D}_{\mathbb{R}^{+}, \mathbb{R}-\mathrm{c}}^{\mathrm{b}}\left(\mathbb{C}_{V}\right)$, we set for short:

$$
\operatorname{THom}\left(F, \mathcal{D} b_{V}\right)=\mathrm{R} \Gamma\left(P ; \operatorname{Thom}\left(j_{!} F, \mathcal{D} b_{P}\right)\right) .
$$

Definition 6.3.1. We denote by $\mathcal{D} b_{V}^{t}$ the conic sheaf associated to the presheaf:

$$
U \mapsto \operatorname{THom}\left(\mathbb{C}_{U}, \mathcal{D} b_{V}\right)
$$

for a subanalytic open cone $U$ in $V$.

The following properties are easily checked.

(i) The conic sheaf $\mathcal{D} b_{V}^{t}$ is conically soft and in particular

$$
H^{j}\left(U ; \mathcal{D} b_{V}^{t}\right)=0 \text { for any } j \neq 0 \text { and any open cone } U \text {. }
$$

(ii) $\mathcal{D} b_{V}^{t}$ is a $D(V \times \bar{V})$-module, where $\bar{V}$ is the complex conjugate of $V$.

(iii) $\mathrm{R} \Gamma\left(V ; \mathcal{D} b_{V}^{t}\right) \simeq \mathrm{THom}\left(\mathbb{C}_{V}, \mathcal{D} b_{V}\right)$.

(iv) $\mathrm{R} \Gamma_{\{0\}}\left(V ; \mathcal{D} b_{V}^{t}\right) \simeq \operatorname{THom}\left(\mathbb{C}_{\{0\}}, \mathcal{D} b_{V}\right)$.

(v) For any open cone $U$, we have in the category of vector spaces

$$
\Gamma\left(U ; \mathcal{D} b_{V}^{t}\right)=\lim _{\longleftarrow} \operatorname{THom}\left(\mathbb{C}_{U^{\prime}}, \mathcal{D} b_{V}\right)
$$


where the projective limit is taken over the subanalytic open cones $U^{\prime} \Subset U$.

Definition 6.3.2. We set:

$$
\mathcal{O}_{V}^{t}=\mathrm{R} \mathcal{H} o m_{D(\bar{V})}\left(\mathcal{O}(\bar{V}), \mathcal{D} b_{V}^{t}\right) .
$$

Proposition 6.3.3. (i) The conic sheaf $\mathcal{O}_{V}^{t}$ is concentrated in degree 0.

(ii) The sheaf $\mathcal{O}_{V}^{t}$ is the conic sheaf associated to the presheaf

$$
U \mapsto \operatorname{THom}\left(\mathbb{C}_{U}, \mathcal{O}_{V}\right)
$$

for a subanalytic convex open cone $U$ in $V$.

(iii) We have

$$
\begin{aligned}
\mathrm{R} \Gamma\left(V ; \mathcal{O}_{V}^{t}\right) & \simeq \operatorname{THom}\left(\mathbb{C}_{V}, \mathcal{O}_{V}\right) \simeq \mathbb{C}[V], \\
\operatorname{R}_{\{0\}}\left(V ; \mathcal{O}_{V}^{t}\right) & \simeq \operatorname{THom}\left(\mathbb{C}_{\{0\}}, \mathcal{O}_{V}\right) \simeq \mathbb{C}\left[V^{*}\right] \otimes \operatorname{det} V[-n] .
\end{aligned}
$$

Here $\mathbb{C}[V]$ denotes the ring of polynomials on $V$.

(iv) Let $U_{1}^{\prime} \Subset U_{1} \Subset U_{2}^{\prime} \Subset U_{2}$ be open cones with $U_{1}^{\prime}$ and $U_{2}^{\prime}$ subanalytic. Then there is a canonical commutative diagram:

$$
\begin{array}{ccc}
\mathrm{R} \Gamma\left(U_{2} ; \mathcal{O}_{V}^{t}\right) & \rightarrow & \mathrm{THom}\left(\mathbb{C}_{U_{2}^{\prime}}, \mathcal{O}_{V}\right) \\
\downarrow & \swarrow & \downarrow \\
\operatorname{R\Gamma }\left(U_{1} ; \mathcal{O}_{V}^{t}\right) & \rightarrow & \mathrm{THom}\left(\mathbb{C}_{U_{1}^{\prime}}, \mathcal{O}_{V}\right) .
\end{array}
$$

Proof. (iii) follows from the corresponding property for $\mathcal{D} b_{V}^{t}$.

(i) By (iii), it is enough to prove this assertion at each point of $V \backslash\{0\}$. Since THom $\left(\mathbb{C}_{U}, \mathcal{O}_{V}\right)$ is concentrated in degree 0 for $U$ convex, this will follow from (iv).

(ii) follows again from (iv).

(iv) Since $\mathcal{D} b_{V}^{t}$ is conically soft, this follows from the commutative diagram:

$$
\begin{array}{ccc}
\Gamma\left(U_{2} ; \mathcal{D} b_{V}^{t}\right) & \rightarrow & \operatorname{THom}\left(\mathbb{C}_{U_{2}^{\prime}}, \mathcal{D} b_{V}\right) \\
\downarrow & \swarrow & \downarrow \\
\Gamma\left(U_{1} ; \mathcal{D} b_{V}^{t}\right) & \rightarrow & \mathrm{THom}\left(\mathbb{C}_{U_{1}^{\prime}}, \mathcal{D} b_{V}\right) .
\end{array}
$$

Corollary 6.3.4. Let $Z_{2} \subset Z_{2}^{\prime} \subset Z_{1} \subset Z_{1}^{\prime}$ be closed cones in $V$ with $Z_{1}^{\prime}$ and $Z_{2}^{\prime}$ subanalytic and $V \backslash Z_{1}^{\prime} \Subset V \backslash Z_{1} \Subset V \backslash Z_{2}^{\prime} \Subset V \backslash Z_{2}$. Then there is a canonical commutative diagram:

$$
\begin{array}{ccc}
\mathrm{R} \Gamma_{Z_{2}}\left(V ; \mathcal{O}_{V}^{t}\right) & \rightarrow & \operatorname{THom}\left(\mathbb{C}_{Z_{2}^{\prime}}, \mathcal{O}_{V}\right) \\
\downarrow & \downarrow & \downarrow \\
\mathrm{R} \Gamma_{Z_{1}}\left(V ; \mathcal{O}_{V}^{t}\right) & \rightarrow & \operatorname{THom}\left(\mathbb{C}_{Z_{1}^{\prime}}, \mathcal{O}_{V}\right) .
\end{array}
$$

Proof. The proof is similar.

Let $V^{*}$ denote the dual vector space. We shall show that the Laplace transform allows us to "quantize" the Fourier-Sato transform. More precisely:

Theorem 6.3.5. (i) The Fourier-Sato transform $\left(\mathcal{O}_{V}^{t}\right)^{\wedge}[n]$ is concentrated in degree 0 .

(ii) The Laplace transform induces an isomorphism $\left(\mathcal{O}_{V}^{t}\right)^{\wedge}[n] \simeq \mathcal{O}_{V^{*}}^{t}$.

(iii) This isomorphism is $D\left(V^{*}\right)$-linear (via the Fourier isomorphism $D(V) \simeq$ $\left.D\left(V^{*}\right)\right)$. 
Proof. (i) Let $U$ be a convex open cone in $V^{*}$, and $Z=U^{\circ} \subset V$ the closed polar cone. By the theory of the Fourier-Sato transform, one knows that:

$$
\mathrm{R} \Gamma\left(U ;\left(\mathcal{O}_{V}^{t}\right)^{\wedge}[n]\right) \simeq \mathrm{R} \Gamma_{Z}\left(V ; \mathcal{O}_{V}^{t}\right)[n] .
$$

Choose open convex cones $U_{1} \Subset U_{2} \Subset U_{3}$ with $U_{2}$ subanalytic. By Proposition 6.3.4 and Theorem 5.2.1, we have a commutative diagram, where $Z_{2}=U_{2}^{\circ}$ :

$$
\begin{aligned}
& \mathrm{R} \Gamma\left(U_{3} ;\left(\mathcal{O}_{V}^{t}\right)^{\wedge}[n]\right) \longrightarrow \\
& \downarrow \\
& \operatorname{THom}\left(\mathbb{C}_{Z_{2}}[-n], \mathcal{O}_{V}^{t}\right) \\
& \mid\urcorner \\
& \operatorname{THom}\left(\mathbb{C}_{U_{2}}, \mathcal{O}_{V^{*}}^{t}\right) .
\end{aligned}
$$

This shows that $\left(\mathcal{O}_{V}^{t}\right)^{\wedge}[n]$ is concentrated in degree 0 on $V \backslash\{0\}$. Moreover, since $\mathrm{R} \Gamma\left(V ;\left(\mathcal{O}_{V}^{t}\right)^{\wedge}[n]\right) \simeq \mathrm{R} \Gamma_{\{0\}}\left(\mathcal{O}_{V}^{t}\right)[n] \simeq \operatorname{THom}\left(\mathbb{C}_{\{0\}}[-n], \mathcal{O}_{V}^{t}\right)$, we see that $\left(\mathcal{O}_{V}^{t}\right)^{\wedge}[n]$ is concentrated in degree 0 , and is isomorphic to the conic sheaf associated with the presheaf $U \mapsto \operatorname{THom}\left(\mathbb{C}_{U}, \mathcal{O}_{V^{*}}\right)$ for an open subanalytic convex cone $U$.

As an application of Theorem 6.3.5, one recovers a result of Brylinski-MalgrangeVerdier [B-M-V] and Hotta-Kashiwara [H-K]. Let $M$ be a finitely generated $D(V)$ module, and denote by $\theta$ the Euler vector field on $V, \theta=\sum_{j} z_{j} \partial_{z_{j}}$. Recall that one says that $M$ is monodromic if $\operatorname{dim}_{\mathbb{C}} \mathbb{C}[\theta] u<\infty$ for any $u \in M$.

Corollary 6.3.6 ([B-M-V], $[\mathrm{H}-\mathrm{K}],[\mathrm{M}])$. Let $M$ be a monodromic $D(V)$-module. Then:

(i) $M^{\vee}$ is monodromic.

(ii) $\mathrm{RH}_{\mathcal{H}} \operatorname{mom}_{D(V)}\left(M, \mathcal{O}_{V}^{t}\right) \stackrel{\sim}{\rightarrow} \mathrm{R} \mathcal{H} \operatorname{om}_{D(V)}\left(M, \mathcal{O}_{V}\right)$.

(iii) $\mathrm{R} \mathcal{H} \operatorname{mom}_{D(V)}\left(M, \mathcal{O}_{V}\right)$ is a conic sheaf (i.e. belongs to $\left.\mathrm{D}_{\mathbb{R}^{+}}^{\mathrm{b}}\left(\mathbb{C}_{V}\right)\right)$.

(iv) The Laplace morphism induces an isomorphism:

$$
\mathrm{R} \mathcal{H} o m_{D(V)}\left(M, \mathcal{O}_{V}\right)^{\wedge}[n] \simeq \mathrm{R} \mathcal{H} o m_{D\left(V^{*}\right)}\left(M^{\vee}, \mathcal{O}_{V^{*}}\right) .
$$

Proof. (i) is obvious

(ii) By standard arguments, one reduces the proof to the case where $M$ has one generator $u$ with the relation $(\theta-\alpha) u=0$. Then the result is clear.

(iii) follows from (ii).

(iv) follows from (ii) and Theorem 6.3.5.

Remark 6.3.7. Corollary 6.3.6 has been recently generalized to non-monodromic $D(V)$-modules regular at infinity by Daia $[\mathrm{D}]$.

For the definition of the functor $\mu$ hom below, we refer to [K-S1].

Corollary 6.3.8. Let $M$ be a monodromic $D(V)$-module, and let $F \in \mathrm{D}_{\mathbb{R}^{+}, \mathbb{R}_{-c}}^{\mathrm{b}}\left(\mathbb{C}_{V}\right)$. Then the Laplace morphism induces an isomorphism of biconic sheaves on $V \times V^{*}$ :

$$
\operatorname{RHom} \operatorname{Hom}_{D(V)}\left(M, \mu h o m\left(F, \mathcal{O}_{V}\right)\right) \simeq \operatorname{R} \mathcal{H o m}_{D\left(V^{*}\right)}\left(M^{\vee}, \mu h o m\left(F^{\wedge}[n], \mathcal{O}_{V^{*}}\right)\right) .
$$

Proof. Apply Corollary 6.3.6 together with [K-S1, Ex. VII 2].

Remark 6.3.9. Let $M: V \rightarrow X$ be a complex vector bundle with fiber dimension $n$. Then all definitions and results, in particular Definition 6.3.2 and Theorem 6.3.5, extend with suitable modifications in this situation. The only "difficulty" is that we need a basis of open cones $U$ such that THom $\left(\mathbb{C}_{U}, \mathcal{O}_{V}\right)$ is concentrated in degree 0 . We may assume that $X$ is open in $\mathbb{C}^{p}$, and $V=X \times \mathbb{C}^{n}$. Then we choose a convex 
$U$ in $X \times \mathbb{C}^{n}$. The fact that $\operatorname{THom}\left(\mathbb{C}_{U}, \mathcal{O}_{V}\right)$ is concentrated in degree 0 in such a case is a well-known result, whose proof may be found, for example, in [D'A-S2].

Remark 6.3.10. See $[\mathrm{M}]$ for the construction of various conic sheaves associated to $\mathcal{O}$ and well suited with Laplace transform.

Remark 6.3.11. One could develop a theory analogous to that of $[\mathrm{S}-\mathrm{K}-\mathrm{K}]$ with $\mathcal{O}$ replaced by the conic sheaf $\mathcal{O}_{V}^{t}$. In particular, one could construct the biconic sheaf of rings $\mathcal{E}_{V}^{t}$ of tempered microdifferential operators on $V \times V^{*}$. This sheaf is invariant by the Fourier transform. Taking its global section, one recovers the Weyl algebra $D(V)$.

6.4. Positive definite matrices. In this section we work in the algebraic setting, following the notations in $\S 4$.

Let $W$ denote the $n(n+1) / 2$-dimensional $\mathbb{C}$-vector space of $n \times n$ symmetric matrices with entries in $\mathbb{C}$. We shall often write an element of $W$ as $a=\left(a_{i j}\right)_{1 \leq i, j \leq n}$. One may identify $W^{*}$ with $W$ by the pairing:

$$
\langle a, b\rangle=\operatorname{tr}(a b)
$$

where $\operatorname{tr}(\cdot)$ is the trace. Let $V=\mathbb{C}^{n}$, endowed with coordinates $z=\left(z_{1}, \ldots, z_{n}\right)$, and consider the morphism:

$$
\begin{aligned}
f: V & \rightarrow W, \\
\left(z_{1}, \ldots, z_{n}\right) & \mapsto\left(z_{i} z_{j}\right)_{1 \leq i, j \leq n} .
\end{aligned}
$$

Notice that this map is finite. If $b \in W^{*}$, we have:

$$
\langle z, b z\rangle=\langle b, f(z)\rangle .
$$

We are interested with the correspondence formally defined by:

$$
\hat{u}(b)=\int_{V} u(z) e^{\langle z, b z\rangle} d z=\int_{W} \int_{V} u(z) e^{\langle a, b\rangle} \delta(a-f(z)) d z d a .
$$

By (6.4.1) this is the composition of the direct image by $f$ and the Laplace transform on $W$ :

$$
V \underset{f}{\longrightarrow} W \swarrow^{W \times W^{*}} \searrow W^{*} .
$$

First, let us calculate $\underline{f}_{*} \mathcal{D}_{V}$. Since $f$ is finite, this module is concentrated in degree 0 . After identifying $\Omega_{V}^{*}$ and $\Omega_{W}$ with $\mathcal{O}_{V}$ and $\mathcal{O}_{W}$ respectively, we have

$$
\underline{f}_{*} \mathcal{D}_{V} \simeq \mathcal{D}_{W} \otimes_{\mathcal{O}_{W}}\left(f_{*} \mathcal{O}_{V}\right) .
$$

Lemma 6.4.1. (i) $f_{*} \mathcal{O}_{V} \simeq \mathcal{L}_{0} \oplus \mathcal{L}_{1}$ where $\mathcal{L}_{0}=\mathcal{O}_{W} u_{0}$, with the defining relations:

$$
\left|\begin{array}{cc}
a_{i k} & a_{i l} \\
a_{j k} & a_{j l}
\end{array}\right| u_{0}=0 \quad \forall i, j, k, l
$$

and $\mathcal{L}_{1}=\sum_{i=1}^{n} \mathcal{O}_{W} u_{1}^{i}$, with the defining relations:

$$
a_{j k} u_{1}^{i}-a_{i k} u_{1}^{j}=0 \quad \forall i, j, k .
$$

(ii) $\mathrm{RHom} \mathcal{O}_{W}\left(f_{*} \mathcal{O}_{V}, \mathcal{O}_{W}\right) \simeq f_{*} \mathcal{O}_{V}[-n(n-1) / 2]$. 
Proof. (i) We leave the tedious calculation to the reader.

(ii) On a complex manifold $X$, denote by $D_{\mathcal{O}}$ the duality functor $D_{\mathcal{O}}(\mathcal{F})=$ $\mathrm{R} \mathcal{H} \operatorname{om}_{\mathcal{O}}\left(\mathcal{F}, \Omega_{X}\right)\left[d_{X}\right]$. Then $f_{*}$ commutes to $D_{\mathcal{O}}$, and we get:

$$
\begin{aligned}
f_{*} \mathcal{O}_{V}[n] & \simeq f_{*} D_{\mathcal{O}}\left(\mathcal{O}_{V}\right) \\
& \simeq D_{\mathcal{O}} f_{*} \mathcal{O}_{V} \\
& \simeq \mathrm{RHom}_{\mathcal{O}_{W}}\left(f_{*} \mathcal{O}_{V}, \mathcal{O}_{W}\right)[n(n+1) / 2]
\end{aligned}
$$

Let $a=\left(a_{i j}\right)_{1 \leq i, j \leq n}$ be a symmetric real matrix. Recall that one says that $a$ is positive semi-definite (resp. positive definite), and one writes $a \geq 0$ (resp. $a>0$ ) if for all $\lambda=\left(\lambda_{1}, \ldots, \lambda_{n}\right) \in \mathbb{R}^{n}$ we have $\sum_{i, j} a_{i j} \lambda_{i} \lambda_{j} \geq 0$ (resp. $>0$, assuming $\lambda \neq 0)$. We introduce the sets:

$$
\begin{aligned}
& Z=\{a \in W ; \operatorname{Im} a=0, \operatorname{Re} a \geq 0\} \\
& \Omega=\left\{b \in W^{*} ;-\operatorname{Re} b>0\right\} .
\end{aligned}
$$

Lemma 6.4.2. One has:

$$
f^{-1} \mathbb{C}_{Z}=\mathbb{C}_{\mathbb{R}^{n}}
$$

Proof. This follows from $f^{-1}(Z)=\mathbb{R}^{n}$.

Lemma 6.4.3. The Fourier-Sato transform induces an isomorphism:

$$
\mathbb{C}_{\Omega}^{\wedge} \simeq \mathbb{C}_{Z}[-n(n+1)] .
$$

Proof. The set $\Omega$ is open and convex in $W^{*}$. The assertion then follows from the well-known fact:

$$
Z=\{a \in W ; \operatorname{tr}(a b) \leq 0 \quad \text { for any } b \in \Omega\}
$$

Let us denote by $N$ the $D\left(W^{*}\right)$-module obtained as the Fourier transform of the $D(W)$-module $\Gamma\left(W ; \underline{f}_{*} \mathcal{D}_{V}\right)$. By Lemma 6.4.1, we have:

$$
\begin{aligned}
N= & N_{0} \oplus N_{1}, \\
N_{0}= & \mathcal{D}_{W^{*}} u_{0} \\
& \text { with relations } \quad\left|\begin{array}{cc}
\partial_{i k} & \partial_{i l} \\
\partial_{j k} & \partial_{j l}
\end{array}\right| u_{0}=0, \quad \forall i, j, k, l, \\
N_{1}= & \sum_{i=1}^{n} \mathcal{D}_{W^{*}} u_{1}^{i} \\
& \text { with relations } \quad \partial_{j k} u_{1}^{i}-\partial_{i k} u_{1}^{j}=0 \quad \forall i, j, k .
\end{aligned}
$$

Here, $\partial_{i j}$ is the restriction to $W^{*}$ of the vector field $\partial_{b_{i j}}+\partial_{b_{j i}}$, as usual.

By Lemma 6.4.1, we have

$$
\mathrm{RHom}_{D\left(W^{*}\right)}\left(N, D\left(W^{*}\right)\right) \simeq N[-n(n-1) / 2] .
$$

Theorem 6.4.4. The correspondence (6.4.2) induces an isomorphism:

$$
\mathcal{S}^{\prime}\left(\mathbb{R}^{n}\right) \simeq \operatorname{Hom}_{D\left(W^{*}\right)}\left(N, H_{[\Omega]}^{0}\left(W^{*} ; \mathcal{O}_{W^{*}}\right)\right) \text {. }
$$

In other words, the correspondence (6.4.2) interchanges the space of tempered distributions on $\mathbb{R}^{n}$ and the space of tempered holomorphic functions on $\Omega$ satisfying the system of differential equations $N$. 
Proof. We have:

$$
\begin{aligned}
\mathcal{S}^{\prime}\left(\mathbb{R}^{n}\right) & \simeq \operatorname{THom}\left(\mathbb{C}_{\mathbb{R}^{n}}[-n], \mathcal{O}_{V}\right) \\
& \simeq \operatorname{THom}\left(f^{-1} \mathbb{C}_{Z}[-n], \Omega_{V}\right) \otimes_{\mathcal{D}} \mathcal{D}_{V} .
\end{aligned}
$$

Applying Proposition 4.2.1, we obtain:

$$
\mathcal{S}^{\prime}\left(\mathbb{R}^{n}\right) \simeq \operatorname{THom}\left(\mathbb{C}_{Z}[-n], \Omega_{W}\right) \otimes_{\mathcal{D}} \underline{f}_{*} \mathcal{D}_{V}
$$

Applying the Laplace isomorphism, we get:

$$
\begin{aligned}
\mathcal{S}^{\prime}\left(\mathbb{R}^{n}\right) & \simeq \operatorname{THom}\left(\mathbb{C}_{\Omega}, \Omega_{W^{*}}\right) \otimes_{D\left(W^{*}\right)}\left(\underline{f}_{*} \mathcal{D}_{V}\right)^{\wedge}[-n(n-1) / 2] \\
& \simeq \operatorname{Hom}_{D\left(W^{*}\right)}\left(N, \operatorname{THom}\left(\mathbb{C}_{\Omega}, \mathcal{O}_{W^{*}}\right)\right) .
\end{aligned}
$$

Here the last isomorphism follows from (6.4.3).

Remark 6.4.5. By the isomorphism in Theorem 6.4.4,

$$
\operatorname{Hom}_{D\left(W^{*}\right)}\left(N_{0}, H_{[\Omega]}^{0}\left(W^{*} ; \mathcal{O}_{W^{*}}\right)\right)
$$

(resp. $\operatorname{Hom}_{D\left(W^{*}\right)}\left(N_{1}, H_{[\Omega]}^{0}\left(W^{*} ; \mathcal{O}_{W^{*}}\right)\right)$ corresponds to the space of even (resp. odd) tempered distributions on $\mathbb{R}^{n}$.

\section{Appendix A. Comparison with the Classical Laplace transform}

In this appendix, we write $\otimes$ instead of $\otimes_{\mathcal{O}}$ for short.

In order to compare our construction with the classical one, we restrict our study to the case of convex cones.

Let $\gamma$ be a closed convex proper subanalytic cone in $V^{*}$. As in $\S 6.1$, we set:

$$
\begin{aligned}
U & =\operatorname{Int} \gamma^{\circ a} \\
& =\{z \in V ; \operatorname{Re}\langle z, w\rangle<0 \text { for all } w \in \gamma\} .
\end{aligned}
$$

Then we have

$$
\left(\mathbb{C}_{U}\right)^{\wedge}[n] \simeq \mathbb{C}_{\gamma}[-n]
$$

We embed $V^{*}$ into the projective space $P^{*}$, and then embed the diagram (5.1.1):

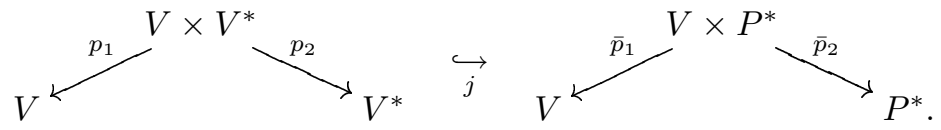

We set

$$
\overline{\mathcal{L}}=\underline{j}_{*} \mathcal{L} \quad \text { and } \quad \overline{\mathcal{L}}^{\prime}=\underline{j}_{*} \mathcal{L}^{\prime}
$$

We set $Z=V \times P^{*}$ and $H=V \times\left(P^{*} \backslash V^{*}\right)$. The partial de Rham complex

$$
0 \rightarrow \mathcal{D}_{Z} \otimes \bigwedge^{n} V \rightarrow \cdots \rightarrow \mathcal{D}_{Z} \otimes V \rightarrow \mathcal{D}_{Z} \rightarrow \underline{p}_{2}^{-1} \mathcal{D}_{P^{*}} \rightarrow 0
$$

gives a resolution of $\underline{\bar{p}}_{2}^{-1} \mathcal{D}_{P^{*}}$, and we obtain a morphism

$$
\underline{\bar{p}}_{2}^{-1} \mathcal{D}_{P^{*}} \rightarrow \mathcal{D}_{Z} \otimes \bigwedge^{n} V[n] \simeq \mathcal{D}_{Z} \otimes \Omega_{V}^{\otimes-1}[n]
$$

We shall construct a similar resolution for $\overline{\mathcal{L}} \otimes \bar{p}_{2}^{-1} \mathcal{D}_{P^{*}}$. Let $\beta: \mathcal{O}_{Z} \otimes V \rightarrow \underline{j}_{*} \mathcal{D}_{Z \backslash H}$ be the morphism $V \ni v \mapsto \partial_{v}+v$. Here $\partial_{v}$ is the vector field on $V$ associated with $v \in V$, regarded as a section of $\mathcal{D}_{Z}$. We also regard $v \in V$ as a meromorphic function on $Z$ with pole in $H$. We set $\mathfrak{h}=\operatorname{Im} \beta \cap \mathcal{D}_{Z}$. Then $\mathfrak{h}$ is a locally free 


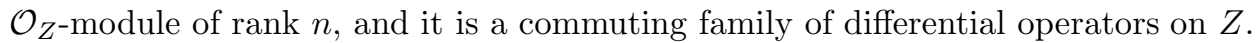
Then the associated Koszul complex gives a sequence:

$$
\begin{aligned}
(\mathrm{A} .2) 0 \rightarrow \mathcal{D}_{Z} \otimes \mathcal{O}_{Z}(H) \otimes \bigwedge^{n} \mathfrak{h} \rightarrow \cdots \rightarrow \mathcal{D}_{Z} \otimes \mathcal{O}_{Z}(H) \otimes \mathfrak{h} & \rightarrow \mathcal{D}_{Z} \otimes \mathcal{O}_{Z}(H) \\
& \rightarrow \overline{\mathcal{L}} \otimes \bar{p}_{2}^{-1} \mathcal{D}_{P^{*}} \rightarrow 0 .
\end{aligned}
$$

Here the last morphism is given by the $\mathcal{O}_{Z}$-linear homomorphisms $\mathcal{O}_{Z}(H) \rightarrow \overline{\mathcal{L}}$ and $\mathcal{O}_{Z} \rightarrow \underline{\bar{p}}_{2}^{-1} \mathcal{D}_{P^{*}}$.

Lemma A.1. (A.2) is an exact sequence.

Proof. It is an exact sequence on $V \times V^{*}$. Let us take the coordinates $\left(z_{1}, \ldots, z_{n}\right)$ of $V$ and the dual coordinates $\left(w_{1}, \ldots, w_{n}\right)$. We shall consider the problem on the open set of $P^{*}$ where $\left(w_{1}^{-1}, w_{2} / w_{1}, \ldots, w_{n} / w_{1}\right)$ is a local coordinate system. Then $\mathfrak{h}$ has a base $w_{1}^{-1} \partial_{z_{1}}+1, \partial_{z_{j}}-w_{1}^{-1} w_{j} \partial_{z_{1}}(2 \leq j \leq n)$. Since their principal symbols form a regular sequence, $0 \rightarrow \mathcal{D}_{Z} \otimes \mathcal{O}_{Z}(H) \otimes \bigwedge^{n} \mathfrak{h} \rightarrow \cdots \rightarrow \mathcal{D}_{Z} \otimes \mathcal{O}_{Z}(H) \otimes \mathfrak{h} \rightarrow$ $\mathcal{D}_{Z} \otimes \mathcal{O}_{Z}(H)$ is an exact sequence. The remaining exactitude is reduced to the statement that

$$
\mathcal{D}_{\mathbb{C}_{2}} / \mathcal{D}_{\mathbb{C}^{2}}\left(x_{1} \partial_{x_{2}}-1\right) \rightarrow\left(\mathcal{D}_{\mathbb{C}^{2}} \exp \left(x_{2} / x_{1}\right)\right)\left[1 / x_{1}\right] \otimes\left(\mathcal{D}_{\mathbb{C}^{2}} / \mathcal{D}_{\mathbb{C}^{2}} \partial_{x_{2}}\right)
$$

is an isomorphism. Here $x_{1}=w_{1}^{-1}, x_{2}=-w_{1}^{-1} \varphi(z, w)$ and the element $1 \in$ $\mathcal{D}_{\mathbb{C}_{2}} / \mathcal{D}_{\mathbb{C}^{2}}\left(x_{1} \partial_{x_{2}}-1\right)$ is sent to $x_{1}^{-1} \exp \left(x_{2} / x_{1}\right)\left[1 / x_{1}\right] \otimes 1$. Now, (A.3) is an isomorphism outside $x_{1} \neq 0$ and both sides of $(\mathrm{A} .3)$ are invariant by $\mathcal{O}_{\mathbb{C}^{2}}\left[1 / x_{1}\right] \otimes \cdot$. Hence (A.3) is an isomorphism.

Since $\operatorname{det} \mathfrak{h}=\mathcal{O}_{Z}(-H) \otimes \operatorname{det} V,\left(\right.$ A.2) gives a morphism in $\mathrm{D}^{\mathrm{b}}\left(\mathcal{D}_{Z}\right)$ :

$$
\begin{aligned}
\overline{\mathcal{L}} \otimes \underline{p}_{2}^{-1} \mathcal{D}_{P^{*}} & \rightarrow \mathcal{D}_{Z} \otimes \mathcal{O}_{Z}(H) \otimes \operatorname{det} \mathfrak{h}[n] \\
& \stackrel{\sim}{\rightarrow} \mathcal{D}_{Z} \otimes \operatorname{det} V[n] \simeq \mathcal{D}_{Z} \otimes \Omega_{V}^{\otimes-1}[n] \\
& \rightarrow \bar{p}_{1}^{-1}\left(\mathcal{D}_{V} \otimes \Omega_{V}^{\otimes-1}\right)[n] .
\end{aligned}
$$

On the other hand, Proposition 5.1.2 implies that $D(V)$ (in fact, more correctly, $\left.\Gamma\left(V ; \mathcal{D}_{V} \otimes \Omega_{V}^{\otimes-1}\right)\right)$ is isomorphic to the module of global sections of the sheaves $\underline{p}_{1 *}\left(\mathcal{L} \otimes \underline{p}_{2}^{-1} \mathcal{D}_{V^{*}}\right) \simeq \underline{\bar{p}}_{1 *}\left(\overline{\mathcal{L}} \otimes \underline{\bar{p}}_{2}^{-1} \mathcal{D}_{P^{*}}\right)$. This isomorphism gives by adjunction

$$
\overline{\mathcal{L}} \otimes \underline{p}_{2}^{-1} \mathcal{D}_{P^{*}} \rightarrow \underline{\bar{p}}_{1}^{-1}\left(\mathcal{D}_{V} \otimes \Omega_{V}^{\otimes-1}\right)[n] .
$$

Lemma A.2. The two morphisms (A.4) and (A.5) are equal (up to a constant multiple).

Proof. In order to prove the lemma it is enough to show that the morphism $\underline{\bar{p}}_{1 *}\left(\mathcal{L} \otimes \underline{\bar{p}}_{2}^{-1} \mathcal{D}_{P^{*}}\right) \rightarrow \mathcal{D}_{V} \otimes \Omega_{V}^{\otimes-1}$ given in Proposition 5.1.2 coincides with the following morphism coming from (A.4) (up to a constant multiple):

$$
\begin{aligned}
\underline{\bar{p}}_{1 *}\left(\mathcal{L} \otimes \underline{\bar{p}}_{2}^{-1} \mathcal{D}_{P^{*}}\right) & \rightarrow \bar{p}_{1 *}\left(\mathcal{D}_{Z} \otimes \Omega_{V}^{\otimes-1}\right)[n] \\
& \rightarrow \underline{p}_{1 *} \underline{\bar{p}}_{1}^{-1}\left(\mathcal{D}_{V} \otimes \Omega_{V}^{\otimes-1}\right)[n] \rightarrow \mathcal{D}_{V} \otimes \Omega_{V}^{\otimes-1} .
\end{aligned}
$$

Since $\underline{\underline{p}}_{1 *}\left(\overline{\mathcal{L}} \otimes \underline{\bar{p}}_{1}^{-1} \mathcal{D}_{P^{*}}\right) \simeq \mathcal{D}_{V} \otimes \Omega_{V}^{\otimes-1}$ and there is only one automorphism of $\mathcal{D}_{V} \otimes \Omega_{V}^{\otimes-1}$ up to a constant multiple, it is enough to show that the morphism (A.6) is an isomorphism. 
We have exact sequences

$$
\begin{aligned}
& 0 \rightarrow \mathcal{O}_{Z} \otimes V^{*} \rightarrow \mathfrak{h}^{*} \rightarrow \mathcal{O}_{H} \rightarrow 0, \\
& 0 \rightarrow \mathcal{O}_{H}(-H) \rightarrow \mathcal{O}_{H} \otimes V^{*} \rightarrow E \rightarrow 0 .
\end{aligned}
$$

Here $E=\left(\mathcal{O}_{H} \otimes V^{*}\right) /\left(\mathfrak{h}^{*}(-H)\right)$ and it is a locally free $\mathcal{O}_{H}$-module of rank $n-1$. Then (A.8) gives an exact sequence

$$
0 \rightarrow \mathcal{O}_{H}(-H) \otimes \bigwedge^{k-1} E \rightarrow \mathcal{O}_{H} \otimes \bigwedge^{k} V^{*} \rightarrow \bigwedge^{k} E \rightarrow 0 .
$$

Recall the well-known vanishing theorem

$$
\bar{p}_{1 *}\left(\mathcal{O}_{H}(-\nu H)\right)=0 \quad \text { for } 0<\nu \leq n .
$$

By induction on $k$, we obtain

$$
\bar{p}_{1 *}\left(\mathcal{O}_{H}(-\nu H) \otimes \bigwedge^{k} E\right)=0 \quad \text { for } \quad 0<\nu, k+\nu \leq n .
$$

The exact sequence (A.7) gives an exact sequence

$$
0 \rightarrow \mathcal{O}_{Z} \otimes \bigwedge^{k} V^{*} \rightarrow \bigwedge^{k} \mathfrak{h}^{*} \rightarrow \bigwedge^{k-1} E \rightarrow 0,
$$

and we have by (A.9):

$$
\bar{p}_{1 *}\left(\mathcal{O}_{Z}(-H) \otimes \bigwedge^{k} \mathfrak{h}^{*}\right)=0 \quad \text { for } \quad 0 \leq k<n .
$$

The remaining case $k=n$ can be calculated as:

$$
\simeq \bar{p}_{1 *}\left(\mathcal{O}_{Z} \otimes \Omega_{V}\right) \simeq \Omega_{V} .
$$

On the other hand, we have for any coherent $\mathcal{O}_{Z}$-module $\mathcal{F}$

$$
\begin{aligned}
\underline{p}_{1 *}\left(\mathcal{D}_{Z} \otimes \mathcal{F}\right) & \simeq \bar{p}_{1 *}\left(\mathcal{D}_{V \leftarrow Z} \otimes \mathcal{F}\right) \\
& \simeq \bar{p}_{1 *}\left(\mathcal{D}_{V} \otimes \Omega_{Z / V} \otimes \mathcal{F}\right) \\
& \simeq \mathcal{D}_{V} \otimes \bar{p}_{1 *} \mathcal{H} \circ \operatorname{Hom}\left(\mathcal{F}, \Omega_{Z / V}\right) \\
& \simeq \mathcal{D}_{V} \otimes \mathcal{H o m}\left(\bar{p}_{1 *} \mathcal{F}^{*}, \mathcal{O}_{V}\right)[-n],
\end{aligned}
$$

where $\mathcal{F}^{*}=\mathcal{H o m}_{\mathcal{O}_{Z}}\left(\mathcal{F}, \mathcal{O}_{Z}\right)$. Hence we have

$$
\begin{aligned}
\underline{\bar{p}}_{1 *}\left(\mathcal{L} \otimes \underline{\bar{p}}_{2}^{-1} \mathcal{D}_{P^{*}}\right) & \simeq \bar{p}_{1 *}\left(\mathcal{D}_{Z} \otimes \mathcal{O}_{Z}(H) \otimes \operatorname{det} \mathfrak{h}\right)[n] \\
& \simeq \mathcal{D}_{V} \otimes \mathcal{H} o m_{\mathcal{D}_{V}}\left(\bar{p}_{1 *}\left(\mathcal{O}_{Z}(-H) \otimes \operatorname{det} \mathfrak{h}^{*}\right), \mathcal{O}_{V}\right) \\
& \simeq \mathcal{D}_{V} \otimes \Omega_{V}^{\otimes-1} .
\end{aligned}
$$

The morphisms (A.1) and (A.4) are related by the commutative diagram:

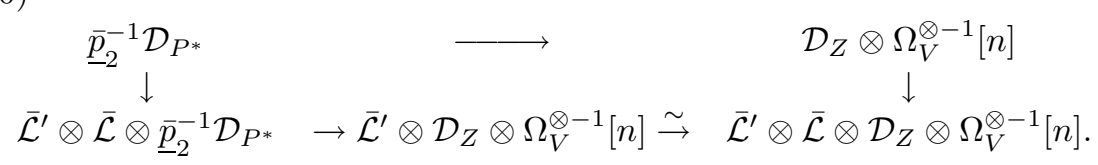


Here the right arrow in the bottom row is given by the morphism $\mathcal{D}_{Z} \otimes \Omega_{V}^{\otimes-1} \rightarrow \overline{\mathcal{L}} \otimes$ $\mathcal{D}_{Z} \otimes \Omega_{V}^{\otimes-1}$ induced by the canonical section of $\mathcal{L}$. We thus obtain the commutative diagram:

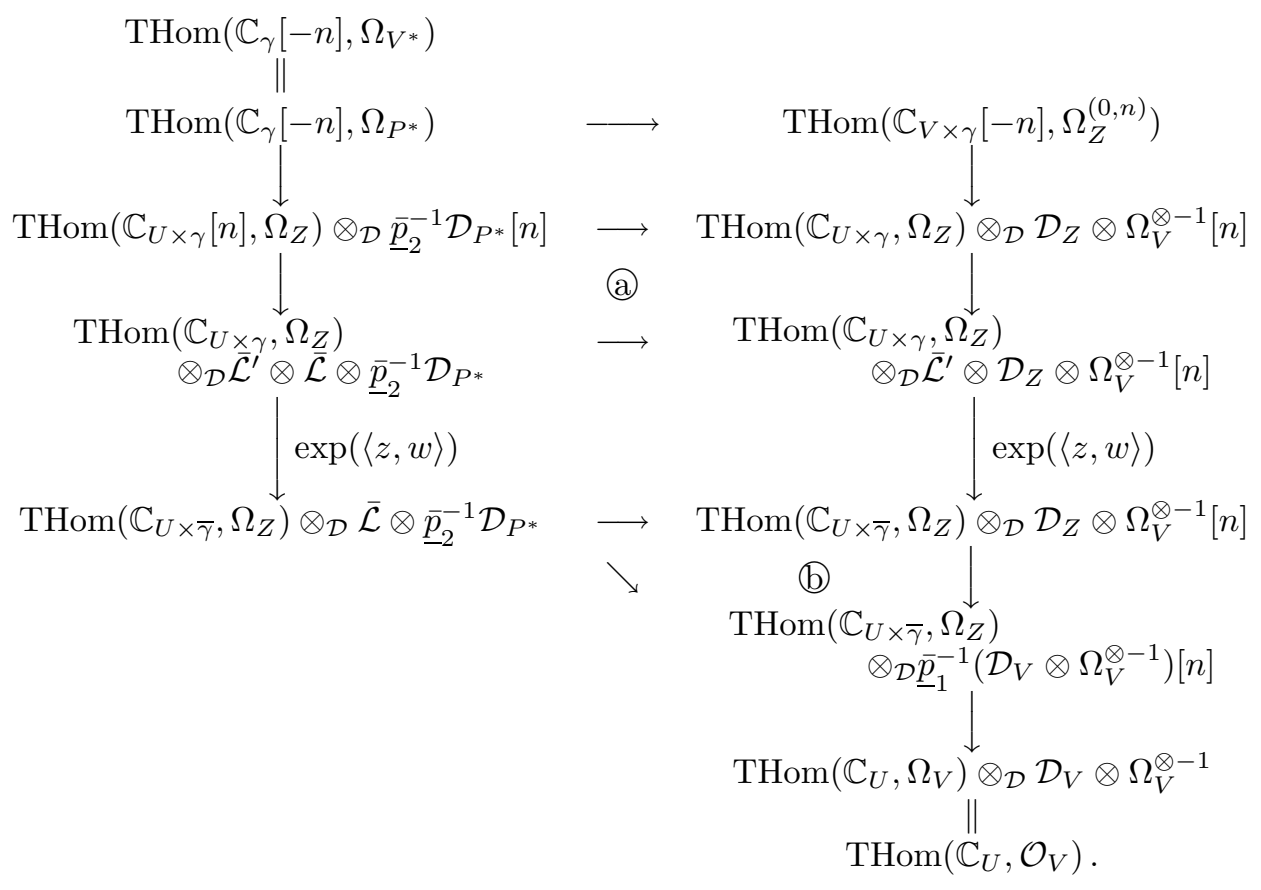

Here $\bar{\gamma}$ is the closure of $\gamma$ in $P^{*}$. The commutativity of @ follows from (A.10) and (b) from Lemma A.2.

The arrows on the left hand side describe the Laplace morphism constructed in §5.2. The arrows on the right hand side define the chain of morphisms:

$$
\begin{array}{rll}
\operatorname{THom}\left(\mathbb{C}_{\gamma}[-n], \Omega_{V^{*}}\right) & \longrightarrow & \operatorname{THom}\left(\mathbb{C}_{U \times \gamma}[-n], \Omega_{V \times P^{*}}^{(0, n)}\right) \\
& \underset{\exp \langle z, w\rangle}{\longrightarrow} & \operatorname{THom}\left(\mathbb{C}_{U \times \bar{\gamma}}[-n], \Omega_{V \times P^{*}}^{(0, n)}\right) \\
& \underset{\int_{P^{*}}}{\longrightarrow} & \operatorname{THom}\left(\mathbb{C}_{U}, \mathcal{O}_{V}\right) .
\end{array}
$$

Hence, the Laplace transform in this paper coincides with the classical Laplace transform

$$
u(w) \mapsto \hat{u}(z)=\int u(w) e^{\langle z, w\rangle} d w .
$$

In particular, if $V_{\mathbb{R}}$ is a real vector space such that $V=\mathbb{C} \otimes_{\mathbb{R}} V_{\mathbb{R}}$, and if $\gamma$ is contained in $V_{\mathbb{R}}^{*}$, then $\operatorname{THom}\left(\mathbb{C}_{\gamma}[-n], \mathcal{O}_{V}\right) \simeq \Gamma_{\gamma}\left(\mathcal{S}^{\prime}\left(V_{\mathbb{R}}^{*}\right)\right)$ is the space of tempered distributions on $V_{\mathbb{R}}$ with support in $\gamma$, and $\mathrm{THom}\left(\mathbb{C}_{U}, \mathcal{O}_{V}\right)$ is the space of tempered holomorphic functions on the convex tube domain $U \subset V$, and we recover the classical Fourier-Laplace transform. 
Proof of Proposition 5.2.3. Decomposing $\sqrt{-1} V_{\mathbb{R}}^{*}$ into proper convex cones $\gamma$, we can reduce the result to the commutative diagram:

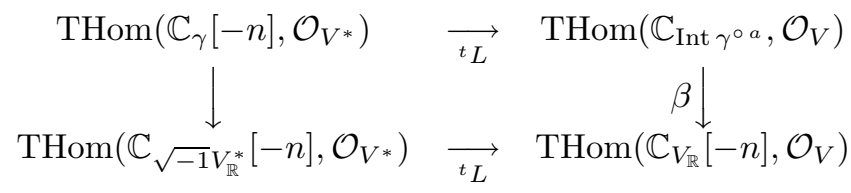

Here $\beta$ is the boundary value morphism.

\section{REFERENCES}

[A] E. Andronikof, Microlocalisation tempérée, Mém. Soc. Math. France, 57 (1994). MR 95e: 58168

[B] J-E. Björk, Analytic D-modules and Applications, Kluwer Academic Publisher, Dordrecht-Boston-London (1993). MR 95f:32014

[B-M-V] J-L. Brylinski, B. Malgrange and J-L. Verdier, Transformation de Fourier géométrique II, C. R. Acad. Sci., 303 (1986), 193-198. MR 88m:58176

[D'A-S1] A. D'Agnolo and P. Schapira, The Radon-Penrose transform for $\mathcal{D}$-modules, J. of Functional Analysis, 139 (1996), 349-382. CMP 96:16

[D'A-S2] A. D'Agnolo and P. Schapira, Leray's quantization of projective duality, Duke Math. J. 84 (1996), 453-496. CMP 96:17

[D] L. Daia, La transformation de Fourier pour les $\mathcal{D}$-modules, Thèse, Université de Grenoble (1995).

[F-G] J. Faraut and S. Gindikin, Private communication to P.S., (1995).

[H-K] R. Hotta and M. Kashiwara, The invariant holonomic systems on a semi-simple Lie algebra, Inventiones Math., 75 (1984), no.2, 327-358. MR 87i:22041

[K] M. Kashiwara, The Riemann-Hilbert problem for holonomic systems, Publ. Res. Inst. Math. Sci., 20 (1984), no.2, 319-365. MR 86j:58142

[K-S1] M. Kashiwara and P. Schapira, Sheaves on manifolds, Grundlehren der Math. Wiss., Springer, 292 (1990). MR 92a:58132; MR 95g:58222

[K-S2] M. Kashiwara and P. Schapira, Moderate and formal cohomology associated with constructible sheaves, Mémoires Soc. Math. France, 64 (1996). CMP 97:04

[K-Sm] M. Kashiwara and W. Schmid, Quasi-equivariant $\mathcal{D}$-modules, equivariant derived category, and representations of reductive Lie groups, in Lie theory and Geometry in honor of Bertram Kostant, Progress of Mathematics, 123 (1994), 457-488. MR 96e:22031

[K-L] N. M. Katz and G. Laumon, Transformation de Fourier et majoration de sommes d'exponentielles, Publ. I.H.E.S., 62 (1985), 361-418. MR 87i:14017

$[\mathrm{M}] \quad$ B. Malgrange, Transformation de Fourier géométrique, Séminaire Bourbaki, 692 (198788). MR 90c: 58178

[Mr] A. Martineau, Distributions et valeurs au bord des fonctions holomorphes, in Proceedings International Summer, Institute Gulbenkian, Lisbon (1964), 195-226, Oeuvres de André Martineau, édition de CNRS (1977), 439-582. MR 36:2833

[S-K-K] M. Sato, T. Kawai and M. Kashiwara, Hyperfunctions and pseudo-differential equations, in Proceedings Katata 1971, Lecture Notes in Math., Springer-Verlag, 287 (1973), 265529. MR 54:8747

Abstract. Let $X \underset{f}{\longleftarrow} \underset{g}{\longrightarrow} Y$ be a correspondence of complex manifolds.

We study integral transforms associated to kernels $\exp (\varphi)$, with $\varphi$ meromorphic on $Z$, acting on formal or moderate cohomologies. Our main application is the Laplace transform. In this case, $X$ is the projective compactification of the vector space $V \simeq \mathbb{C}^{n}, Y$ is its dual space, $Z=X \times Y$ and $\varphi(z, w)=\langle z, w\rangle$. We obtain the isomorphisms:

$$
F \stackrel{W}{\otimes} \mathcal{O}_{V} \simeq F^{\wedge}[n] \stackrel{W}{\otimes} \mathcal{O}_{V^{*}}, \quad \operatorname{THom}\left(F, \mathcal{O}_{V}\right) \simeq \operatorname{THom}\left(F^{\wedge}[n], \mathcal{O}_{V^{*}}\right)
$$


where $F$ is a conic and $\mathbb{R}$-constructible sheaf on $V$ and $F^{\wedge}$ is its Fourier-Sato transform. Some applications are discussed.

RIMS, KYOTO UNIVERSITY, KYOTO 606-01, JAPAN

Institut de Mathématiques, Université Paris Vi, Case 82, 4 Pl Jussieu, 75252 Paris, FRANCE

E-mail address: schapira@math.jussieu.fr 